\title{
ELECTRON-BEAM-INDUCED RADIO EMISSION FROM ULTRACOOL DWARFS
}

\author{
S. Yu ${ }^{1}$, J. G. Doyle ${ }^{1}$, A. Kuznetsov ${ }^{1,2}$, G. Hallinan ${ }^{3}$, A. Antonova ${ }^{4}$, A. L. MacKinnon ${ }^{5}$, And A. Golden ${ }^{6}$ \\ ${ }^{1}$ Armagh Observatory, College Hill, Armagh BT61 9DG, UK; syu@arm.ac.uk \\ ${ }^{2}$ Institute of Solar-Terrestrial Physics, Irkutsk 664033, Russia \\ ${ }^{3}$ California Institute of Technology, 1200 E. California Blvd., Pasadena, CA 91125, USA \\ ${ }^{4}$ Department of Astronomy, St. Kliment Ohridski University of Sofia, 5 James Bourchier Blvd., 1164 Sofia, Bulgaria \\ ${ }^{5}$ School of Physics and Astronomy, University of Glasgow, Glasgow G12 8QQ, UK \\ ${ }^{6}$ Price Center, Albert Einstein College of Medicine, Yeshiva University, Bronx, NY 10461, USA \\ Received 2011 December 14; accepted 2012 April 4; published 2012 May 25
}

\begin{abstract}
We present the numerical simulations for an electron-beam-driven and loss-cone-driven electron-cyclotron maser (ECM) with different plasma parameters and different magnetic field strengths for a relatively small region and short timescale in an attempt to interpret the recent discovered intense radio emission from ultracool dwarfs. We find that a large amount of electromagnetic (EM) field energy can be effectively released from the beam-driven ECM, which rapidly heats the surrounding plasma. A rapidly developed high-energy tail of electrons in velocity space (resulting from the heating process of the ECM) may produce the radio continuum depending on the initial strength of the external magnetic field and the electron beam current. Both significant linear polarization and circular polarization of EM waves can be obtained from the simulations. The spectral energy distributions of the simulated radio waves show that harmonics may appear from 10 to $70 v_{\mathrm{pe}}$ ( $v_{\mathrm{pe}}$ is the electron plasma frequency) in the non-relativistic case and from 10 to $600 v_{\text {pe }}$ in the relativistic case, which makes it difficult to find the fundamental cyclotron frequency in the observed radio frequencies. A wide frequency band should therefore be covered by future radio observations.
\end{abstract}

Key words: brown dwarfs - magnetic fields - masers - polarization - radio continuum: stars - stars: low-mass

Online-only material: color figures

\section{INTRODUCTION}

Ultracool dwarfs (UCDs) are those objects with spectral type later than M7 and low luminosity. Recent observations of 193 UCDs reveal that 12 UCDs produce intense radio emission with flux densities up to hundreds of $\mu \mathrm{Jy}$ (e.g., Berger et al. 2001, 2005; Berger 2002; Burgasser \& Putman 2005; Hallinan et al. 2006; Antonova et al. 2008; McLean et al. 2012). Some of them show a highly circularly polarized radio pulse with regular periods and a flux density up to $15 \mathrm{mJy}$ (Hallinan et al. 2007; Berger et al. 2009).

In a range of past studies, these radio features of UCDs have been presented as a function of magnetic field, spectral type, rotation, age, binarity, and association with the X-ray and H $\alpha$ emission (e.g., Berger et al. 2005, 2010; Hallinan et al. 2006; Antonova et al. 2008; McLean et al. 2012). The regular periods of radio pulses from TVLM 513-46546 and the L dwarf binary 2MASSW J0746425+200032 indicate that the radio activity of UCDs is strongly in conjunction with their rotation (Hallinan et al. 2007; Berger et al. 2009), which is one of the crucial factors to influence the magnetic field by a differential-rotation-induced dynamo theory (Parker 1955) although other mechanisms, such as a turbulence-induced dynamo (Durney et al. 1993) (for smallscale fields) or $\alpha^{2}$ dynamo (Chabrier \& Küker 2006) (for largescale fields), can also contribute to the magnetic field. The topology of the magnetic field on UCDs may be understood as a dipole due to the narrow bunching of multiple pulses of both left and right 100\% polarization (Hallinan et al. 2007). Berger et al. (2009), however, have suggested that the field topology maybe more complex-due to a $1 / 4$ phase lag of the radio pulses compared to $\mathrm{H} \alpha$. This is based on the assumption that the emission is parallel to the magnetic field, but if the emission is perpendicular to the field, then the $1 / 4$ phase lag is in agreement with a dipole magnetic field geometry. Hence, a determination of the magnetic field and its structure is critically important.

Precise analysis of the time domain of radio emission from TVLM 513-46546 (Doyle et al. 2010) and sporadic radio emission from UCDs (Antonova et al. 2007) show that largescale fields may be stable on UCDs for long periods, from a few months to years. The steady magnetic fields on UCDs are also confirmed by the multi-frequency observations of a late-M dwarf binary (Osten et al. 2009). The field strength can be determined using two specific radiation mechanisms - gyrosynchrotron or electron-cyclotron maser (ECM). The first mechanism suggests a field strength in the range of 0.1-1000 G (Berger 2002, 2006), while the latter implies a $\mathrm{kG}$ field. However, the form of the frequency-field-strength relation becomes complicated when the ECM mechanism is applied to a many-electron system since (1) the absorption and emission of different layers in the magnetosphere (or atmosphere) would be significant due to the different plasma environments and magnetic field configuration (e.g., see the discussion of the gyromagnetic absorption in Melrose \& Dulk 1982); and (2) as we will show in this paper, for the motion of a group of electrons in a magnetic field, the ECM can generate a multiple peak structure for the spectral energy distribution (SED). Hence, coverage of the full dynamic radio spectrum, including the low-frequency band (hundreds of $\mathrm{MHz}$ ) and the very high frequency band, is important for a proper understanding of the radiation process.

The observed power-law radio continuum and low-level circular polarization $(<40 \%)$ from several UCDs, such as for the M8.5 dwarf DENIS 1048-3956 between 3 and $30 \mathrm{GHz}$ in four 2 GHz bandwidths (Ravi et al. 2011), may be interpreted as gyrosynchrotron radiation if the surrounding plasma is optically thin. On the other hand, the high brightness temperature $\left(\sim 10^{15} \mathrm{~K}\right)$ and highly (up to $\left.100 \%\right)$ circular polarization of the radio pulses (Hallinan et al. 2007; Berger et al. 2009) suggest 
that the dominant emission mechanism is the ECM. This mechanism was initially assumed to be driven by a loss-cone velocity distribution (Melrose \& Dulk 1982, and references therein) but was subsequently developed to ring shell distribution or horseshoe distribution (Pritchett \& Strangeway 1985).

The operation of the ECM is rather simple, i.e., electrons with an anisotropic distribution transversely move in an external magnetic field. This leads to the application of the ECM to the radio emission from the solar planets, magnetic-chemically peculiar stars (e.g., Lo et al. 2012), and some compact extragalactic radio sources (e.g., Melrose \& Dulk 1982; Dulk 1985; Treumann 2006, and references therein). The generation of the auroral kilometric radiation on the Earth has been interpreted in terms of the ECM, where the velocity distribution of electrons may not be a loss cone caused by the magnetic mirror effect, but due to a horseshoe distribution associated with the acceleration of particles in a magnetic-field-aligned electric field (Wu \& Lee 1979; Chiu \& Schulz 1978; Ergun et al. 2000). A similar interpretation can be applied to the decametric radiation on Jupiter, Saturnian kilometric radiation (Zarka 1998, 2004), and solar millisecond microwave spikes (Aschwanden 1990b; Fleishman et al. 2003). The ECM can also be a strong candidate for the possible presence of radio emission in exoplanets (Zarka 2007; Grießmeier et al. 2007; Jardine \& Cameron 2008). Furthermore, it can be an effective mechanism for the radio-frequency heating of X-ray-emitting plasma in solar flares (Melrose \& Dulk 1984). Recently, it was suggested that the ECM generated by the lowdensity relativistic plasma in many fine localized regions can interpret the high brightness temperature detected from Blazar jets (Begelman et al. 2005). More discussion and application of the ECM can be seen in a review in Treumann (2006).

In fact, gyrosynchrotron radiation and ECM belong to the same family - the motion of electrons in a magnetic field. ECM may efficiently heat the surrounding electrons to form a highenergy tail or even a bump in the velocity space that induces gyrosynchrotron radiation to contribute to the radio continuum. Combining the short timescale and self-quenching features of the ECM, we can also understand the high brightness temperature of the radio pulses. Electron beams would be common in the context of the astrophysical process since there are plenty of sources for generating them. Recent cool atmospheric models indicate that collisions of significant volume of molecular clouds may trigger a tempestuous discharge process such as lightning, resulting in a high-degree ionization in the local molecules or atoms, and the release of a large number of electrons (Helling et al. 2011), which increases the probability of magnetic reconnection events. The electrons may be released and accelerated from the magnetic reconnection or outflow jets indicated by oxygen forbidden emission lines (Whelan et al. 2007), which might result from the intense activity below the chromosphere of UCDs.

In order to understand the radio emission from UCDs and infer the magnetic field and the plasma environment, an investigation of a many-electron system moving in an external magnetic field is essential. Numerical simulations can provide the opportunity to obtain the detailed process self-consistently and an interpretation for the radio emission. In this paper, we attempt to interpret the radio pulses from UCDs using an electron-beam (or current-beam) driven ECM, with concentration on the microscopic energy transformation by treating the electron population as charged particles in a simulation box. We also investigate the growth rate and polarization of the released electromagnetic (EM) waves and the SED. In Section 2, we briefly describe the physical model, numerical method, and the initial conditions to carry out the simulations. In Sections 3 and 4, we present the results for the non-relativistic beam-driven and loss-cone-driven ECM. In Section 5, we present the results for the relativistic beam-driven instability. We make a brief comparison with the observations in Section 6 and then summarize the simulations and draw conclusions in Section 7.

\section{CONFIGURATION OF SIMULATION}

\subsection{Physical Model}

We assume that electron beams are generated by some intense events on UCDs, e.g., magnetic reconnection or jet events. When the electron beams move to the magnetosphere of the UCDs, they interact with the magnetic field and the surrounding plasma. In the present study, we neglect the influence of heavy ions. Here, we investigate the energy transfer, including the induced EM field energy, the drift kinetic energy, and thermal kinetic energy of electrons, plus the growth rate and polarization of the EM fields and the SED.

We start the simulations from the fundamental physical laws. The EM fields and the interaction between them and electrons can be described by Maxwell's equations, i.e., Ampère's law, Faraday's law of induction, Gaussian's law for magnetism, Gaussian's law, and the definition of current:

$$
\begin{gathered}
\nabla \times \mathbf{B}=\mu_{0} \mathbf{J}+\frac{1}{c^{2}} \frac{\partial \mathbf{E}}{\partial t}, \\
\nabla \times \mathbf{E}=-\frac{\partial \mathbf{B}}{\partial t}, \\
\nabla \cdot \mathbf{B}=0, \\
\nabla \cdot \mathbf{E}=\frac{\rho}{\epsilon_{0}}, \\
\nabla \cdot \mathbf{J}=-\frac{\partial \rho}{\partial t},
\end{gathered}
$$

where $\mathbf{B}$ and $\mathbf{E}$ are the magnetic field and electric field, respectively, $\mathbf{J}$ is the current, $t$ is the time, $c$ is the speed of light, $\rho$ is the charge density, $\epsilon_{0}$ is the permittivity, and $\mu_{0}$ is the permeability.

The motion of electrons is governed by the Lorentz force, which can be written as

$$
q(\mathbf{E}+\mathbf{v} \times \mathbf{B})=\frac{\mathrm{d} m \mathbf{v}}{\mathrm{d} t},
$$

where $\mathbf{v}$ is the velocity of one individual particle, $q$ is the charge of a single particle (here it is for an electron), and $m$ is the electron mass. In the non-relativistic case, we have $m=m_{\mathrm{e}}$, where $m_{\mathrm{e}}$ is the rest mass of the electron. In the relativistic case, we have $m=\gamma m_{\mathrm{e}}$, where $\gamma=1 /\left(\sqrt{1-(\mathbf{v} / c)^{2}}\right)$ is the Lorentz factor. The relativistic case is also a general case for the motion equation of particles.

\subsection{Initial Configurations of the Simulations}

These equations were solved self-consistently as a pure initial value problem using a particle-in-cell method in a twodimensional space ( $x$ - and $y$-directions) and three velocity and field dimensions $(x$-, $y$-, $z$-directions $)$. Some of the numerical methods used here are from Omura \& Matsumoto (1993) and Omura (2005). The Buneman-Boris method was used to solve the equation of motion (Hockney \& Eastwood 1981; Birdsall \& 


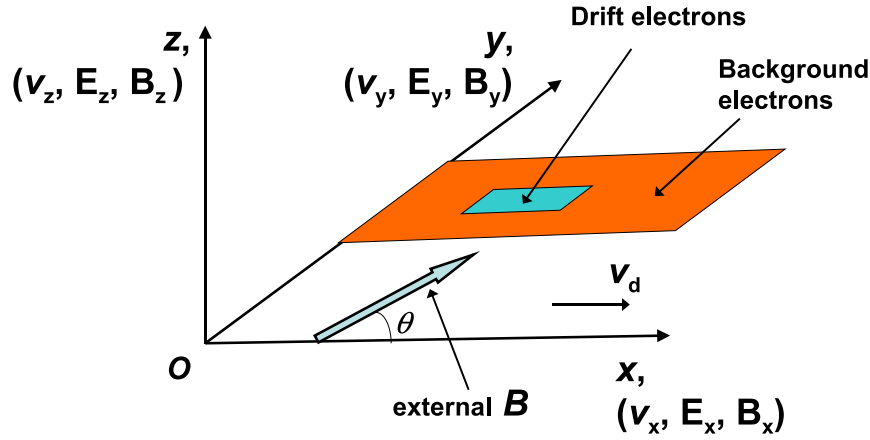

Figure 1. Schematic diagram to present the simulation box for the radio emission from ultracool dwarfs. In an orthogonal reference system with spatial directions $x, y$, and $z, v_{x}, v_{y}$, and $v_{z}$ represent the velocity components of particles, while $E_{x}, E_{y}, E_{z}, B_{x}, B_{y}$, and $B_{z}$ represent the electric and magnetic field components.

(A color version of this figure is available in the online journal.)

Table 1

Reference Values of Electron Densities and Related Parameters

\begin{tabular}{lccc}
\hline \hline $\begin{array}{l}n_{\mathrm{e}} \\
\left(\mathrm{cm}^{-3}\right)\end{array}$ & $\begin{array}{c}v_{\mathrm{pe}} \\
\left(\mathrm{s}^{-1}\right)\end{array}$ & $\begin{array}{c}\lambda_{\mathrm{D}} \\
(\mathrm{cm})\end{array}$ & $\begin{array}{c}v_{\text {th }} / \text { Temperature } \\
\left(\mathrm{cm} \mathrm{s}^{-1} / \mathrm{K}\right)\end{array}$ \\
\hline $10^{12}$ & $8.98 \times 10^{9}$ & 0.005 & $3 \times 10^{8} / 3 \times 10^{5}$ \\
$10^{10}$ & $8.98 \times 10^{8}$ & 0.05 & $3 \times 10^{8} / 3 \times 10^{5}$ \\
$10^{8}$ & $8.98 \times 10^{7}$ & 0.5 & $3 \times 10^{8} / 3 \times 10^{5}$ \\
$10^{6}$ & $8.98 \times 10^{6}$ & 5 & $3 \times 10^{8} / 3 \times 10^{5}$ \\
$10^{4}$ & $8.98 \times 10^{5}$ & 50 & $3 \times 10^{8} / 3 \times 10^{5}$ \\
$10^{2}$ & $8.98 \times 10^{4}$ & 500 & $3 \times 10^{8} / 3 \times 10^{5}$ \\
\hline
\end{tabular}

Langdon 1985). The equation of continuity of charge was solved by a charge conservation method (Villasenor \& Buneman 1992). The spacing grid for the EM field in the present simulations is $64 \times 64$. The time step in each simulation is $\Delta t=0.001 v_{\mathrm{pe}}^{-1}$, where $v_{\mathrm{pe}}$ is the electron plasma frequency, while the space step is $\Delta x=\Delta y=0.125 \lambda_{\mathrm{D}}$, where $\lambda_{\mathrm{D}}=v_{\mathrm{th}} /\left(2 \pi v_{\mathrm{pe}}\right)$ is the Debye length, with $v_{\text {th }}$ being the thermal velocity of the electrons. All the velocities in the simulations are normalized by the speed of light $c$. The charge-mass ratio of electrons is assumed to be -1 . We use an open boundary in the simulated system. This simulation configuration and the intrinsic properties of the electrons do not vary in any of the simulations.

In each of the simulations, we assume that a constant external magnetic field $\mathbf{B}_{0}$ exists in the spatial $x-y$-plane, and the angle between $\mathbf{B}_{0}$ and $x$-direction is defined as $\theta$ with $0^{\circ} \leqslant \theta \leqslant 90^{\circ}$. The charged particles are initially distributed in the $x-y$-plane randomly. We assume that background thermal electrons may exist in the radio emission region, i.e., the magnetosphere of UCDs, with number density $n_{\text {th }}$ and thermal velocity $v_{\text {th }}$. The injected electrons have a number density $n_{\mathrm{d}}$, thermal velocity $v_{\text {th }}^{\prime}$, and drift velocity $\mathbf{v}_{\mathrm{d}}$ along the $x$-direction. Figure 1 shows the spatial simulation box schematically. In the simulations, we determine the strength of the external magnetic field via its close relation with the cyclotron frequency $v_{\mathrm{ce}} \approx 2.8 B_{0} \mathrm{MHz}$. The relation between plasma frequency and the number of electrons is $v_{\mathrm{pe}} \approx 8.98 \times 10^{-3}\left(n_{\mathrm{e}} \mathrm{cm}^{-3}\right)^{1 / 2} \mathrm{MHz}$.

Since we do not know the electron density in the radio emission region on UCDs, a range of values and related plasma parameters are listed in Table 1. From the values in the table, we see that the present simulations are in a relatively microregion and short timescale $(0.1 \mathrm{~ns}$ to $10 \mu \mathrm{s})$.

In order to see the influence of the above parameters on the released EM waves, we set a group of standard values for
Table 2

Various Parameters in the Simulations (Section 3) and Their Values

\begin{tabular}{|c|c|c|c|}
\hline Parameters & Symbols & $\begin{array}{l}\text { Standard } \\
\text { Values }\end{array}$ & $\begin{array}{l}\text { Optional } \\
\text { Values }\end{array}$ \\
\hline Plasma frequency & $v_{\mathrm{pe}}$ & 1 & $\ldots$ \\
\hline Cyclotron frequency & $v_{\mathrm{ce}}$ & 10 & 0,5 \\
\hline Angle: $\mathbf{B}_{0}$ and $\mathbf{v}_{\mathrm{d}}$ & $\theta$ & $90^{\circ}$ & $0,45^{\circ}$ \\
\hline $\begin{array}{l}\text { Thermal velocity } \\
\text { of background } e\end{array}$ & $v_{\text {th }}$ & $0.01 c$ & $0.005 c, 0.05 c$ \\
\hline $\begin{array}{l}\text { Thermal velocity } \\
\text { of drift } e\end{array}$ & $v_{\mathrm{th}}^{\prime}$ & $0.01 c$ & $0.005 c, 0.05 c$ \\
\hline Drift velocity & $\mathbf{v}_{\mathrm{d}}$ & $0.05 c$ & $0.0 c, 0.1 c$ \\
\hline $\begin{array}{l}\text { Number of superparticles } \\
\text { of background } e\end{array}$ & $n_{\mathrm{th}}$ & 16384 & 0,32768 \\
\hline $\begin{array}{l}\text { Number of superparticles } \\
\text { of drift } e\end{array}$ & $n_{\mathrm{d}}$ & 16384 & $\cdots$ \\
\hline
\end{tabular}

Note. Note that part of the values may vary in Sections 4 and 5.

them (see Table 2). In this model, we assume the direction of the external magnetic field parallel to the $y$-direction. The cyclotron frequency is set to be 10 times the plasma frequency. We take the thermal velocity of the background electrons and the drift electrons as $0.01 c$. This means that the temperature of the electrons is about $3 \times 10^{5} \mathrm{~K}$, determined by $T=(1 / 2) m_{\mathrm{e}} v_{\mathrm{th}}^{2} / k$ in the non-relativistic case, where $k$ is the Boltzmann constant. We take $v_{\mathrm{d}}=0.05 c$. We vary the value for one of the parameters, while the other parameters remain as the standard values. These parameters and their values are summarized in Table 2 . We will interpret these parameters in Section 3.2. The standard values of these parameters are derived from estimations of solar bursts (typically $0.1 c$ to $0.5 c$ for the drift velocity and $0.002 c$ to $0.05 c$ for the thermal velocity; Dulk 1985) and the studies on auroral kilometric radiation on the Earth, Jovian millisecond bursts, and Saturnian kilometric radiation $(\sim 1-10 \mathrm{keV}$ for the energetic electrons and $\sim 100 \mathrm{eV}$ for the thermal electrons; Zarka 1998; Hess et al. 2007a, 2007b; Zarka 2007; Lamy et al. 2010). The values of the electron velocities in the relativistic case (see Section 5) refer to the work of Louarn et al. (1986), and references therein. We choose the optional values over a wide range so that the approximate functions between energies and the parameters can be obtained.

In this paper, we distinguish the irregular thermal motion of the electrons and their uniform motion. The thermal energy of the electrons in the non-relativistic case is defined and calculated from the thermal motion of the electrons by

$$
E_{\mathrm{th}}=E_{\mathrm{tk}}-E_{\mathrm{d}}=\sum_{i=1}^{n}\left(\frac{1}{2} m_{\mathrm{e}} \mathbf{v}_{i}^{2}\right)-\frac{1}{2} m_{\mathrm{e}}\left(\sum_{i=1}^{n} \mathbf{v}_{i}\right)^{2},
$$

where $n$ is $n_{\text {th }}$ for the background electrons and $n_{\mathrm{d}}$ for the drift electrons, $m_{\mathrm{e}}$ is the electron mass, and $\mathbf{v}_{i}$ is the velocity of the $i$ th particle. On the right-hand side of this equation, the first term represents the total kinetic energy of the system $E_{\mathrm{tk}}$, and the second term describes the drift energy of the electrons $E_{\mathrm{d}}$.

In the relativistic case, the energy of one electron is defined by the energy-momentum relation,

$$
E_{i \mathrm{e}}^{2}=\mathbf{p}_{i \mathrm{e}}^{2} c^{2}+m_{\mathrm{e}}^{2} c^{4}=\left(\gamma m_{\mathrm{e}} c^{2}\right)^{2},
$$

where $\mathbf{p}_{i \mathrm{e}}=\gamma m_{\mathrm{e}} \mathbf{v}_{i}$ is the momentum of the $i$ th electron and $\gamma=1 /\left(\sqrt{1-\left(\mathbf{v}_{i} / c\right)^{2}}\right)$ is the Lorentz factor. Therefore, the kinetic energy of one electron can be expressed as

$$
E_{i \mathrm{ek}}=E_{i \mathrm{e}}-m_{\mathrm{e}} c^{2}=(\gamma-1) m_{\mathrm{e}} c^{2} .
$$


Then the total kinetic energy of the system $E_{\mathrm{tk}}$ is

$$
E_{\mathrm{tk}}=\sum_{i=1}^{n} E_{i \mathrm{ek}}
$$

For the drift energy of the system $E_{\mathrm{d}}$, we first define $\gamma_{\mathrm{d}}=$ $1 /\left(\sqrt{1-\left(\sum_{i=1}^{n} \mathbf{v}_{i} / n c\right)^{2}}\right)$. Then we have

$$
E_{\mathrm{d}}=\left(\gamma_{\mathrm{d}}-1\right) \cdot n \cdot m_{\mathrm{e}} c^{2}
$$

So the thermal energy is $E_{\mathrm{th}}=E_{\mathrm{tk}}-E_{\mathrm{d}}$. The definition of the drift energy of the system in both the non-relativistic and relativistic cases realizes the uniform motion of the system along one direction via eliminating the irregular random motion of the electrons. Under these definitions, the non-relativistic case (i.e., Equation (7)) is a good approximation of the relativistic case (i.e., Equations (8)-(11)) when particles move with low velocities compared to the speed of light. Our calculations indicate that the equations for the relativistic case are valid for the velocity range $v_{i} \in[0, c)$, while the non-relativistic case is only valid for low-velocity particles. A general definition of temperature then becomes $k T=(\gamma-1) m_{\mathrm{e}} c^{2}$.

The energy of the EM field $w$ can be described by the Poynting theorem, so that we have $w=(1 / 2) \epsilon_{0} \mathbf{E}^{2}+\left(\mathbf{B}^{2} / 2 \mu_{0}\right)$, where we take $\epsilon_{0}=1$ and $\mu_{0}=\left(1 / c^{2} \epsilon_{0}\right)$. The first term on the right-hand side of this equation gives the electric field energy, while the second term represents the magnetic field energy. All kinds of energies are normalized by the initial total energy of the system to satisfy energy conservation. We exclude the energy of the external magnetic field $\mathbf{B}_{0}$.

The growth rate is defined as

$$
\Gamma=\frac{\ln \left(E_{(t+\Delta t)}^{2}\right)-\ln \left(E_{(t)}^{2}\right)}{2 \Delta t} .
$$

The degree of linear polarization in the EM fields $\Pi$ is

$$
\Pi=\frac{w_{\perp}-w_{\|}}{w_{\perp}+w_{\|}}
$$

where $w_{\perp}$ is the EM field energy in the direction perpendicular to the external magnetic field and $w_{\|}$is the EM field energy in the direction parallel to the external magnetic field.

The background electrons are assumed to be in thermal equilibrium, so their velocity distribution obeys a Gaussian distribution. In this paper it is always taken as the following expression:

$$
f_{0 \mathrm{~b}}=n_{\mathrm{th}} \exp \left(-\frac{v_{x}^{2}+v_{y}^{2}+v_{z}^{2}}{v_{\mathrm{th}}^{2}}\right) \text {, }
$$

where $v_{x}, v_{y}, v_{z}$ are the components of the velocity $\mathbf{v}_{\mathrm{b}}$ of the background electrons along the $x$-, $y$-, $z$ - directions, respectively.

The injected electrons have an intrinsic thermal velocity distribution, but due to the acceleration, they will obtain a drift velocity along some direction. For the purpose of simplicity, we assume that all of the injected electrons are accelerated along one direction. We take the direction as the $x$-direction in our simulations, so that the initial velocity distribution for the injected electrons can be expressed as

$$
f_{0 \mathrm{~d}}=n_{\mathrm{d}}\left(\frac{v_{x}^{\prime}-v_{\mathrm{d}}}{v_{\mathrm{th}}^{\prime}}\right)^{2 l} \exp \left(-\frac{\left(v_{x}^{\prime}-v_{\mathrm{d}}\right)^{2}+v_{y}^{\prime 2}+v_{z}^{\prime 2}}{v_{\mathrm{th}}^{\prime 2}}\right),
$$

where $l=0,1,2,3, \cdots$, and $v_{x}^{\prime}, v_{y}^{\prime}, v_{z}^{\prime}$ are the components of the velocity $\mathbf{v}_{\text {in }}$ of the injected electrons along the $x-, y-, z-$ directions, respectively. $l$ is the parameter that describes the size of the loss cone in velocity space. In the present simulations, we take $l=0$ and 3 . All the particles are assumed to be randomly distributed in space.

\section{RESULTS I: $l=0$}

When $l=0$, Equation (15) becomes a Gaussian distribution. In this section, we investigate the influence of the injected electrons with the Gaussian velocity distribution on the evolution of the space and velocity distribution of the electrons, the energy conversion efficiency, the transfer between drift kinetic energy and thermal energy of the system, and the growth rate and polarization of the released EM waves.

\subsection{Standard Model for Beam-driven ECM}

We first present the simulation with standard parameters in detail; this we call the standard model. This standard configuration means that the background electrons and the injected electrons have the same temperature $\left(10^{5} \mathrm{~K}\right)$ but the density of the latter is higher. (Note that we use the definition of the temperature in Section 2.2, which reflects the measure of the velocity dispersion). This is for the purpose of modeling a dense electron beam injected into the magnetosphere of a UCD.

Figure 2 shows the spatial evolution of the electrons (a movie is available for the spatial evolution of the system at http://www.arm.ac.uk/highlights/2012/600/beam/beam_space/). The time in each snapshot is $t=0, t=0.3, t=1.04, t=2.86$, $t=4.9, t=6.52 v_{\mathrm{pe}}^{-1}$ from the top left to bottom right. Due to the existence of the external magnetic field, the motion of the electrons along the $x$-direction is confined and they can only freely move along the $y$-direction, which is parallel to the magnetic field. The motion of the injected electrons as a whole should be in a helical orbit with the radius determined by $r_{\mathrm{d}}=\left(m_{\mathrm{e}} v_{\mathrm{d}}\right) / q B$ since the induced magnetic field is very small. In this simulation, $r_{\mathrm{d}}$ is about 0.715 . Since we only perform a two-dimensional simulation, we see the oscillation of the injected electrons in the $x-y$-plane instead of a helical motion.

The mix of background electrons and injected electrons in the velocity space triggered by the EM field is rather interesting. Figure 3 shows snapshots of the velocity distribution of the electrons at the same time as in Figure 2 (a movie is available for the velocity evolution at http://www.arm. ac.uk/highlights/2012/600/beam/beam_velocity/). As seen in the top left panel in the figure, the velocities of both background electrons and injected electrons are initially a Gaussian distribution, with the injected electrons having a drift velocity of $0.05 c$. When time evolves, the current generated by the injected electrons induces a strong electric field along the direction perpendicular to the external $\mathbf{B}_{0}$, which only alters the direction of the flow. This electric field accelerates a fraction of the background electrons so that a tail at high velocity is developed in $v_{x}$ and $v_{z}$ (see the velocity distribution in each time snapshot), while the injected electrons are decelerated by the electric field, losing their drift velocity along the $x$-direction gradually and wavily. Also because of the perpendicular electric field, the injected electrons gain a drift velocity in $v_{z}$ that oscillates between $-0.1 c$ and $0.1 c$. The appearance of a double peak in the distribution of $v_{y}$ is due to the magnetic constraint and the acceleration of the induced electric field on the perpendicular velocity $v_{x}-v_{y}$. One can imagine that if the evolutionary time 


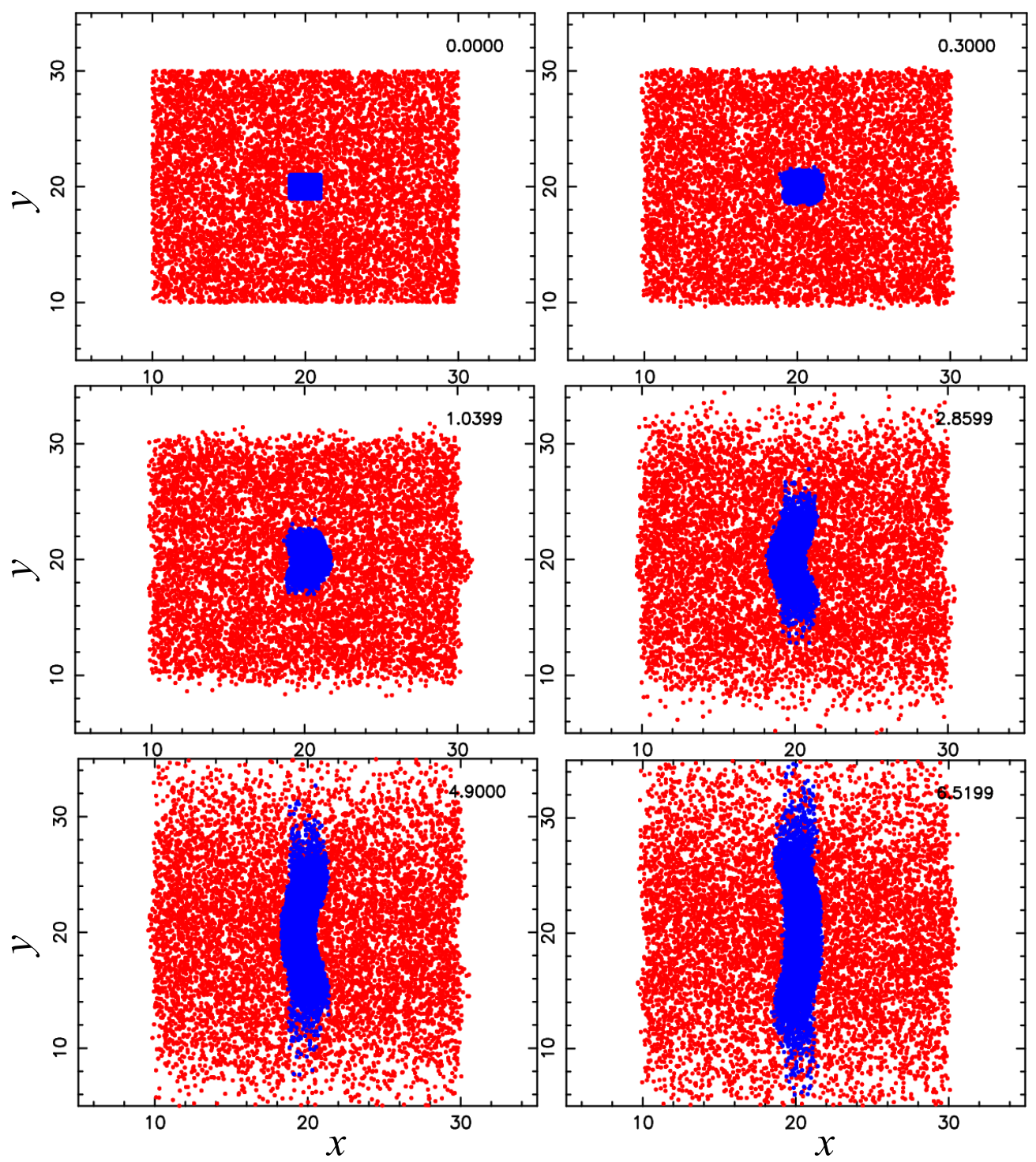

Figure 2. History of the distribution of the background electrons (red points) and beam electrons (blue points) in spatial $x-y$-plane. Different panels from top left to bottom right represent the snapshots for time $t=0.00,0.30,1.04,2.86,4.90$, and $6.52 v_{\mathrm{pe}}^{-1}$.

(A color version of this figure is available in the online journal.)

is sufficiently long, the electrons may separate into two groups. One group has a velocity along the direction of the external $\mathbf{B}_{0}$, while the other group has the opposite velocity direction.

The consequence of this process is that the velocities of the electrons evolve from a concentrated Gaussian distribution to an expanded quasi-Gaussian distribution with a high-velocity tail. During the diffusion process of the particles in velocity space, an energy transfer occurs between the kinetic energy of the electrons and the induced EM field energy. As the injected electrons move in the external $\mathbf{B}_{0}$ at time $t=0$, they start releasing their kinetic energy to the EM wave energy in the manner of an increase in the induced EM field strength. After some time, the EM field energy will reach its maximum while $E_{\mathrm{tk}}$ approaches a minimum. Then the EM field energy may be absorbed by the electrons to compensate for lost kinetic energy. The $E_{\mathrm{tk}}$ will increase after a short time as the field energy decreases. Oscillations in $E_{\mathrm{tk}}$ and the field energy will last for some time until the system is balanced and there is an anti-phase relation between the two kinds of energies.
Figure 4 illustrates the evolution of the total kinetic energy $E_{\mathrm{tk}}$, drift energy $E_{\mathrm{d}}$, and thermal energy $E_{\mathrm{th}}$ of the electrons and the field energy $w$. We plot the same time points for the space and velocity distribution of the electrons in this figure as solid black circles.

As we can see from this figure, $E_{\mathrm{tk}}$ and $w$ are exactly antiphase as expected. The multi-peaks of $w$ should be associated with the different wave mode in frequency space, which will be shown later. The relation of the fine structure of $E_{\mathrm{d}}$ and $E_{\mathrm{th}}$ is not as obvious as the relation between $E_{\mathrm{tk}}$ and $w$ since the interaction between $E_{\mathrm{d}}$ and $E_{\mathrm{th}}$ is via the EM field as a time and space delay can affect the phase relation. However, we see that $E_{\mathrm{d}}$ decreases dramatically at the starting time when $E_{\mathrm{th}}$ increases rapidly. After about $1.5 v_{\mathrm{pe}}^{-1}$, when $E_{\mathrm{d}}$ and $E_{\mathrm{th}}$ have approximately equal values, the decrease of $E_{\mathrm{d}}$ slows, and so does the increase in $E_{\mathrm{th}}$. At time $7.1 v_{\mathrm{pe}}^{-1}$, at least $70 \%$ of $E_{\mathrm{d}}$ is converted to $E_{\mathrm{th}}$. This is interesting because it means that the transverse motion of electrons in an external magnetic field may be an efficient way to heat the ambient electrons. 

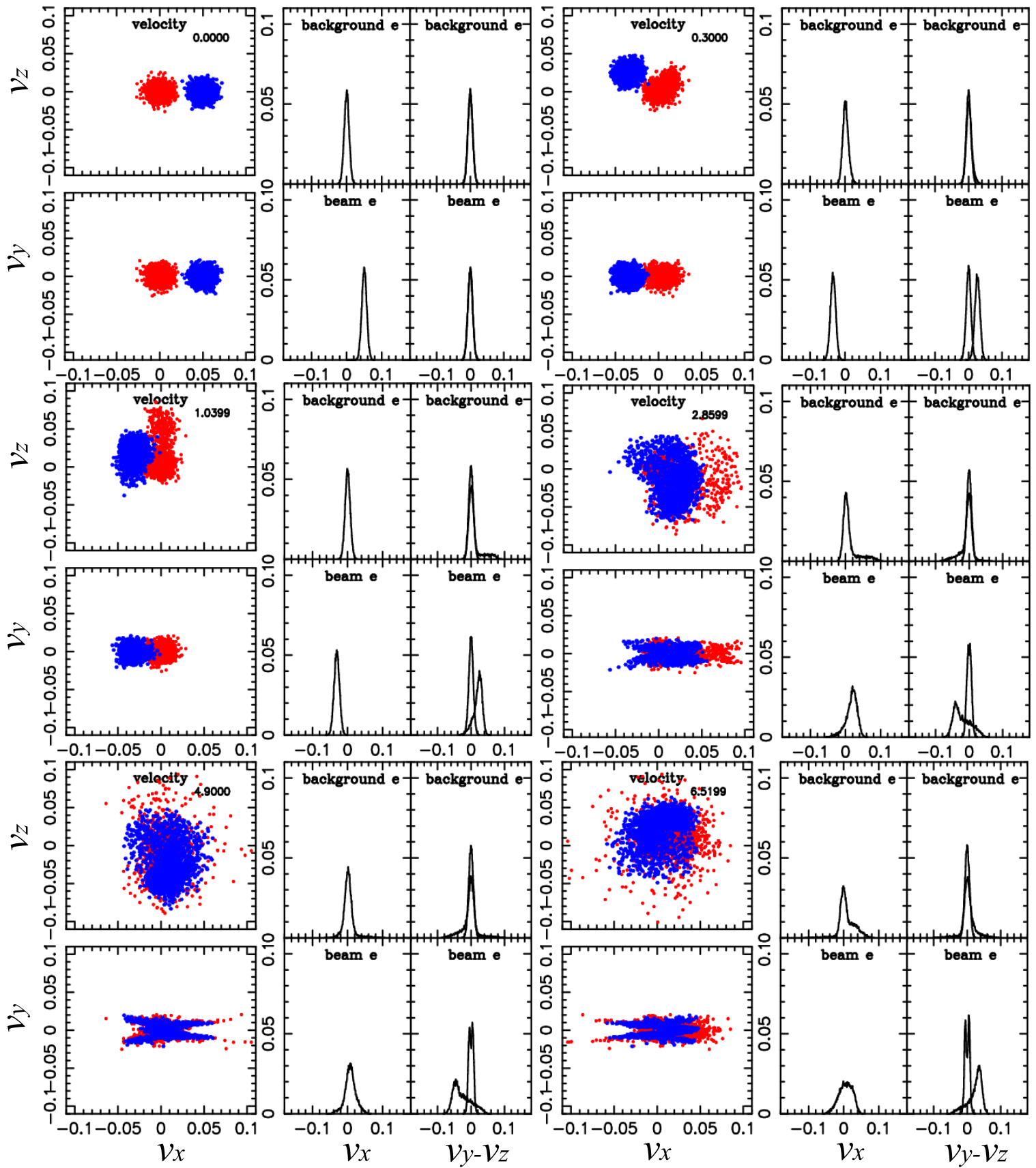

Figure 3. History of the velocity distribution of the background electrons (red points) and beam electrons (blue points). Different panels from top left to bottom right represent the snapshots for time $t=0.00,0.30,1.04,2.86,4.90$, and $6.52 v_{\mathrm{pe}}^{-1}$.

(A color version of this figure is available in the online journal.)

The maximum growth rate of the EM wave in this simulation is about $9.68 \times 10^{2} v_{\text {pe. }}$. The polarization of the released EM waves is highly linear or circular, depending on the initial configuration. More details on the growth rate and polarization in the standard model will be shown in the next section. Figure 5 shows the evolution history of the EM wave energy perpendicular and parallel to the external magnetic field. Again, we use the solid black circles to denote the time points for the space and velocity distribution of the electrons shown in Figures 2 and 3. From this figure, we clearly see that most of the EM waves are polarized in the direction perpendicular to $\mathbf{B}_{0}$, while only a very small fraction of the EM waves are released parallel to $\mathbf{B}_{0}\left(\sim 10^{-3}\right.$ of the perpendicular energy).

\subsection{Influences of Parameters}

In this section, we investigate the influence of the following parameters on the energy history, growth rate, and polarization of the EM waves:

1. The strength of the external magnetic field $B_{0} . B_{0}$ can be determined by the cyclotron frequency,

$$
B_{0} \approx 0.357 \times 10^{3} \frac{\nu_{\mathrm{ce}}}{\mathrm{GHz}} \mathrm{G} .
$$

To date, all detected radio emission of UCDs is in the $\mathrm{GHz}$ band, while observations performed with the NRAO very large array in 2007 show no trace of radio emission from 

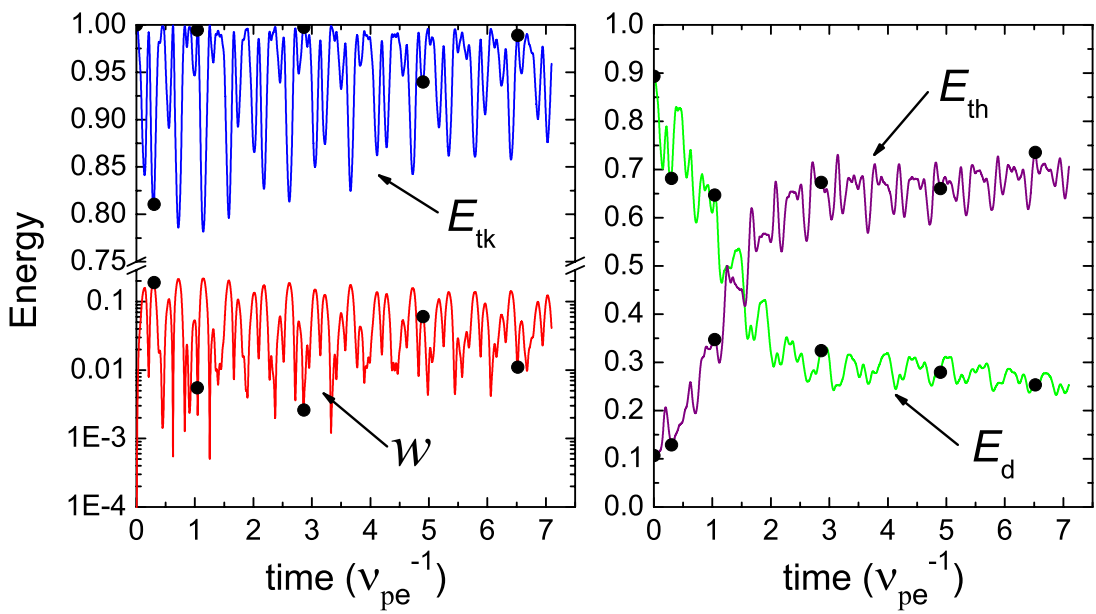

Figure 4. Energy history of the standard model. Blue line in the left panel denotes the total kinetic energy, and red line stands for the field energy. In the right panel, purple line is for the total thermal energy of the system, and green line is for the drift energy of the injected electrons. Black points denote the time as in Figures 2 and 3.

(A color version of this figure is available in the online journal.)

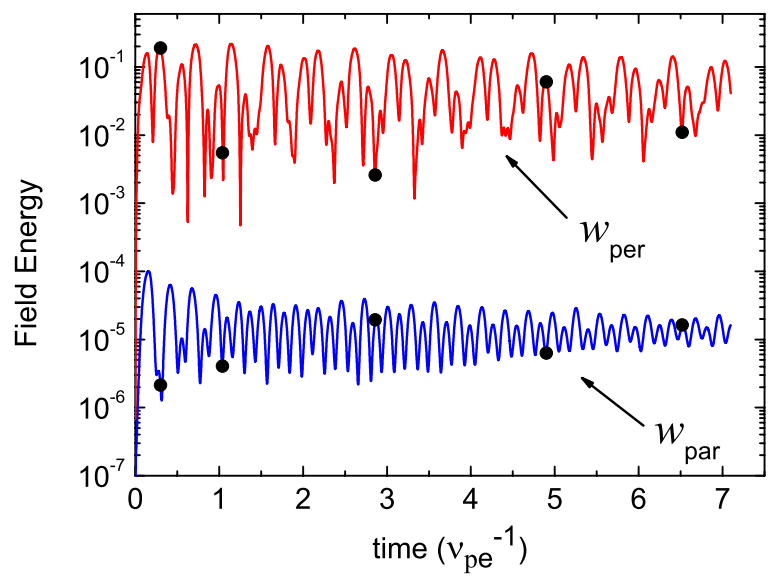

Figure 5. History of the field energy along the direction of perpendicular (red line) and parallel (blue line) to the external magnetic field. Black points denote the time as in Figures 2 and 3.

(A color version of this figure is available in the online journal.)

two UCDs at $325 \mathrm{MHz}$, placing an upper flux limit of $\sim 900 \mu \mathrm{Jy}$ at the $2.5 \sigma$ level (Jaeger et al. 2011). From this, we infer that the magnitude of $B_{0}$ is a few kilo gauss (if the radio emission is truly at the cyclotron frequency). Here, we take $v_{\mathrm{ce}} / v_{\mathrm{pe}}=0,5$, and 10 to see how the magnetic field can affect the simulation results. When $v_{\mathrm{ce}} / v_{\mathrm{pe}}=0$, there is no external magnetic field.

2. The angle $\theta$ between the magnetic field and the drift velocity. $\theta$ is one of the crucial parameters to influence the direction of the radiated EM waves, i.e., the polarization. When $\theta=0^{\circ}$, the injected electrons move uniformly parallel to the external $\mathbf{B}_{0}$ in addition to the irregular thermal motion. When $\theta=90^{\circ}$, the motion of the injected electrons is perpendicular to $\mathbf{B}_{0}$.

3. The drift velocity $v_{d}$. We expect that the radio emission may be enhanced by increasing the value of $v_{d}$. In this section, we take $v_{\mathrm{d}}=0 c, 0.005 c$, and $0.01 c$ to avoid the relativistic effect where gyrosychrotron emission plays an important role. When $v_{\mathrm{d}}=0 c$, we see the effect of pure thermal electrons moving in the external $\mathbf{B}_{0}$ on the induced EM waves.
4. The temperature $T$. We investigate the response of the radiated EM waves and the transfer between the energies by varying $v_{\text {th }}$. We take $v_{\text {th }}=0.005 c, 0.01 c$, and $0.05 c$, for which the corresponding $T$ is $7.5 \times 10^{4}, 3 \times 10^{5}$, and $7.5 \times 10^{6} \mathrm{~K}$.

5. The background electrons by changing $n_{\mathrm{th}} / n_{\mathrm{d}}$. Since our computation capability is limited, we only investigate the effect of the existence of the background electrons on the EM waves and the energy transport. We take $n_{\text {th }} / n_{\mathrm{d}}=$ $0,1,2$. When $n_{\text {th }} / n_{\mathrm{d}}=0$, there are no background electrons.

Figure 6 shows the energy evolution in each simulation. We see that the EM field energy (left panels) is in a very low level in all three specific cases (see the colored lines). In the first case, there is no magnetic field (which can be understood as the injected electrons just passing by very quickly without sufficient energy transfer via the EM field). In the second case, the motion of the injected electrons is parallel to the external magnetic field. In this case, the induced electrons cannot remain within the simulation box since the magnetic field cannot constrain them. In the third case, the drift velocity of the injected electrons is $0 c$; although the injected electrons can remain and interact with the background, there is no coherent current, i.e., the field energy is still small although higher than in the other two cases.

A common expression of these cases is $\mathbf{v} \times \mathbf{B}_{0}=0$, which is the Lorentz force induced by the external magnetic field. This force makes the initially coherent current bend (i.e., the electrons with drift velocity), leading to spatial curled EM fields that are the medium to accomplish energy transport among different kinds of energies.

In other cases except the above three cases, the field energy can maintain a much higher level after they reach the maximum, typically orders of 2-3 that of the above cases. As shown in Figure 6, the field energies in all cases oscillate with large amplitude caused by the transfer between the kinetic energy and field energy. In other words, these oscillations reflect the emission and absorption of electrons to the EM waves. With the achievement of the diffusion process of the electrons in the velocity space, a dynamic balance is approached. This results in a gradual decrease in the amplitudes of the oscillations with time. The time for relaxation of the field energy is $>10 v_{\mathrm{pe}}^{-1}$ 

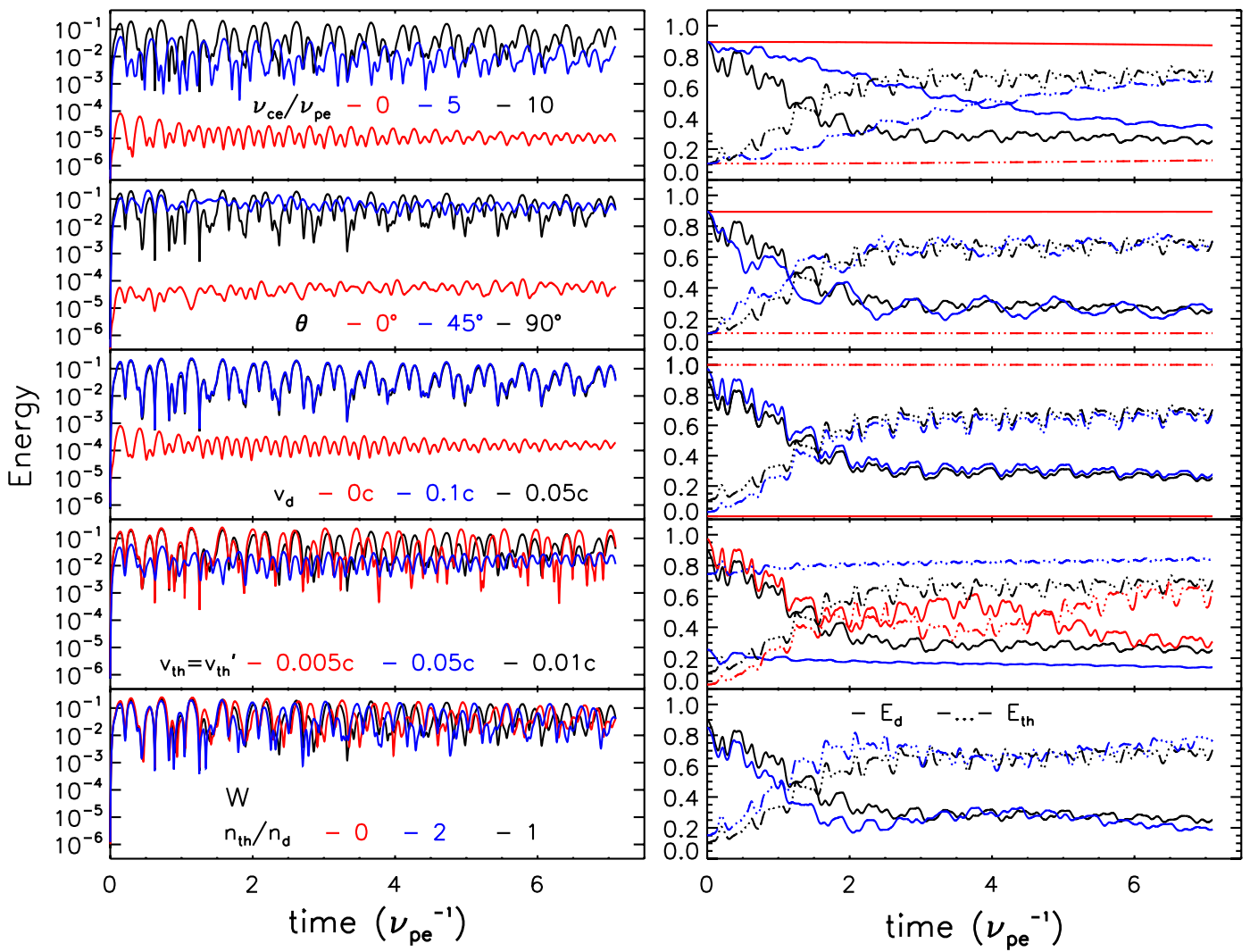

Figure 6. Evolution of the field energy (left panels), and total thermal energy and the drift energy (right panels). Different panels are for different parameters, i.e., first row: $v_{\mathrm{ce}} / v_{\mathrm{pe}}=0$ (red line), 5 (blue line), 10 (black line); second row: $\theta=0^{\circ}$ (red line), $45^{\circ}$ (blue line), $90^{\circ}$ (black line); third row: $v_{\mathrm{d}}=0 c$ (red line), $0.1 c$ (blue line), $0.05 c$ (black line); fourth row: $v_{\mathrm{th}}=0.005 c$ (red line), $0.05 c$ (blue line), $0.01 c$ (black line); and fifth row: $n_{\mathrm{th}} / n_{\mathrm{d}}=0$ (red line), 2 (green line), 1 (black line). In the right panels, the solid line is the drift energy while the dot-dot-dot-dashed line is for the thermal energy. Note that the black colored lines in this figure are always for the model with standard parameters.

(A color version of this figure is available in the online journal.)

which is much longer than the time taken for the field energy to reach maximum, $\sim 0.14 v_{\mathrm{pe}}^{-1}$.

As expected, increasing $B_{0}$ by a factor of two, i.e., $v_{\mathrm{ce}} / v_{\mathrm{pe}}$ from 5 to 10 , leads to a rise in the field energy. It seems that the mean value of the field energy is not sensitive to $\theta$ when $\theta=45^{\circ}$ and $90^{\circ}$. However, the modes (or direction) of the EM waves are affected. In the case of $\theta=45^{\circ}$, the EM waves have a similar energy level in the direction perpendicular and parallel to $\mathbf{B}_{0}$, which is shown in Figure 7 where we discuss polarization. The increase of $v_{d}$ from $0.05 c$ to $0.1 c$ only raises the energy level slightly in the present simulations. In fact, when $v_{d}$ is sufficiently high, we have to consider the relativistic effect and hence gyrosynchrotron radiation, which will be addressed in Section 5 . The mean energy level of the EM waves is not sensitive to the thermal velocity and background electron number density. Note that in the standard model, the initial density of the background electrons is $\sim 100$ times less than that of the injected electrons.

The polarization of the EM waves, or their energy distribution with respect to the magnetic field direction, is shown in Figure 7 quantitatively. The EM waves in the standard model are $100 \%$ linearly polarized. When $\mathbf{v} \times \mathbf{B}_{0}=0$, the EM waves frequently switch their direction from parallel-dominant to perpendicular-dominant, and rarely do they have up to $50 \%$ linear polarization. This behavior indicates that the waves have a significant linearly polarized component. In addition to $\theta$, thermal motion (i.e., temperature) of the electrons can influence the level of polarization. As we see from the bottom second panel in Figure 7, the electrons with temperature $7.5 \times 10^{6} \mathrm{~K}$ can generate the EM waves with $\sim 50 \%-90 \%$ linear polarization, while $100 \%$ linearly polarized waves are generated by the electrons with temperature $7.5 \times 10^{4}$ and $3 \times 10^{5} \mathrm{~K}$. A low density of background electrons does not alter the highly linear polarization in the present simulations.

The dissipation of the drift energy in the injected electrons (when they move in the external magnetic field) is important because this may be sufficient to increase the thermal energy of the system. We show the history of the drift energy and thermal energy in Figure 6. Comparing the right panels in Figure 6, we find that (1) when $\mathbf{v} \times \mathbf{B}_{0}=0$, the energy transfer is the least efficient. In this case, there is almost no energy exchange; (2) when initially $\mathbf{v} \times \mathbf{B}_{0} \neq 0$ and $E_{\mathrm{d}}>E_{\mathrm{th}}, E_{\mathrm{d}}$ can be transported to $E_{\text {th }}$ rapidly; the timescale in which $E_{\mathrm{d}}$ and $E_{\text {th }}$ reach the same value is about $\sim 1-5 v_{\mathrm{pe}}^{-1}$.

Figure 8 illustrates the growth rate of the EM waves in the simulations. The increase of the system temperature $(3 \times$ $10^{5}-7.5 \times 10^{6} \mathrm{~K}$ ) will suppress the growth rate significantly. The number of the electrons also affects the growth rate, but their relation is not so obvious.

\subsection{Spectrum}

The EM field (wave) energy is the integral (or sum if the signal is discrete) of the contribution from different frequencies. In order to obtain the energy distribution of the EM field in frequency space (i.e., the SED), we perform a fast Fourier transform to the EM field energy history. Figure 9 illustrates the SED. 


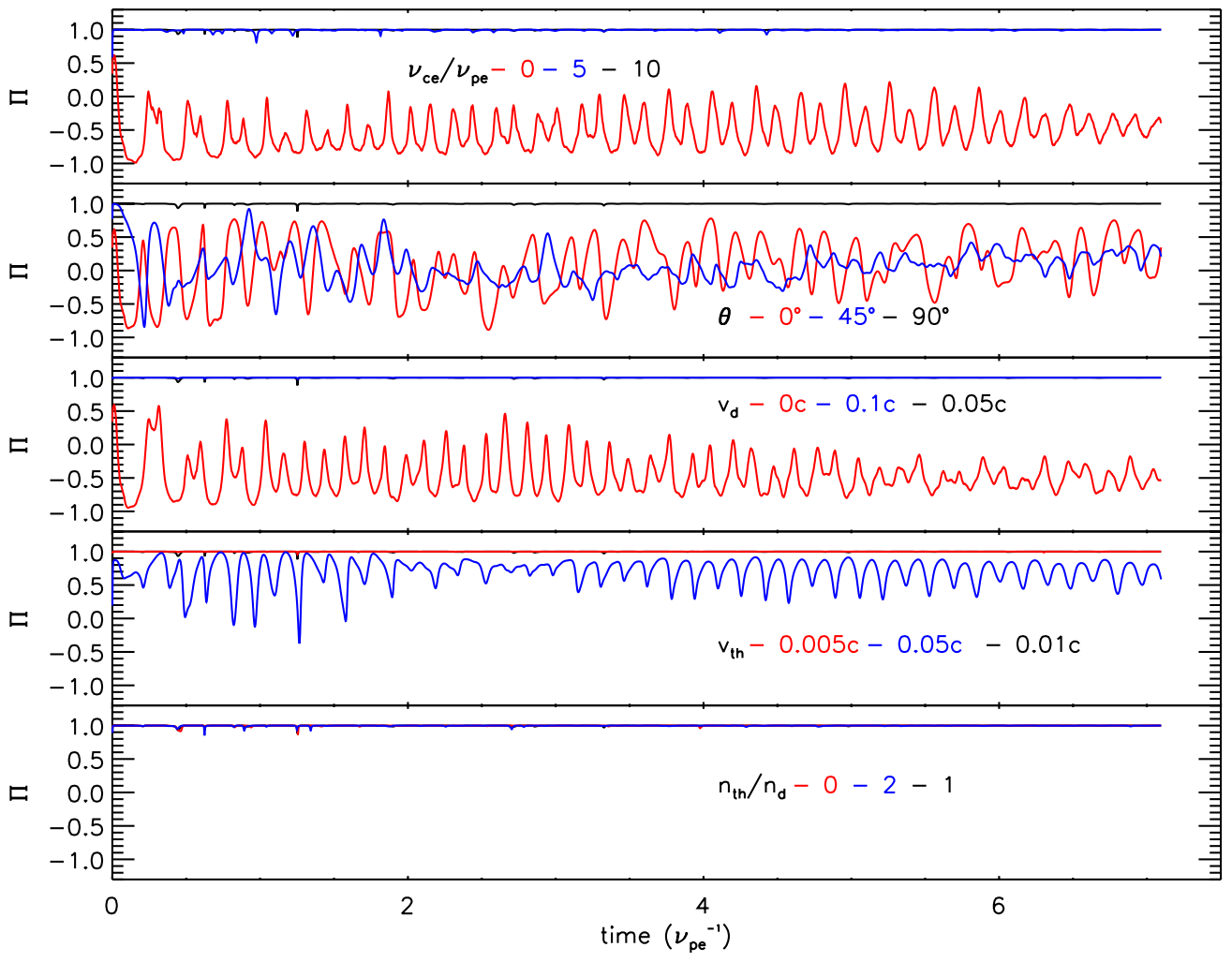

Figure 7. Time evolution of the degree of linear polarization in the simulations. Each panel is for one parameter, i.e., from top to bottom, $v_{\mathrm{ce}} / v_{\mathrm{pe}}, \theta, v_{\mathrm{d}}, v_{\mathrm{th}}, n_{\mathrm{th}} / n_{\mathrm{d}}$. Different colors in each panel are for different values of the parameters as shown in the panel.

(A color version of this figure is available in the online journal.)

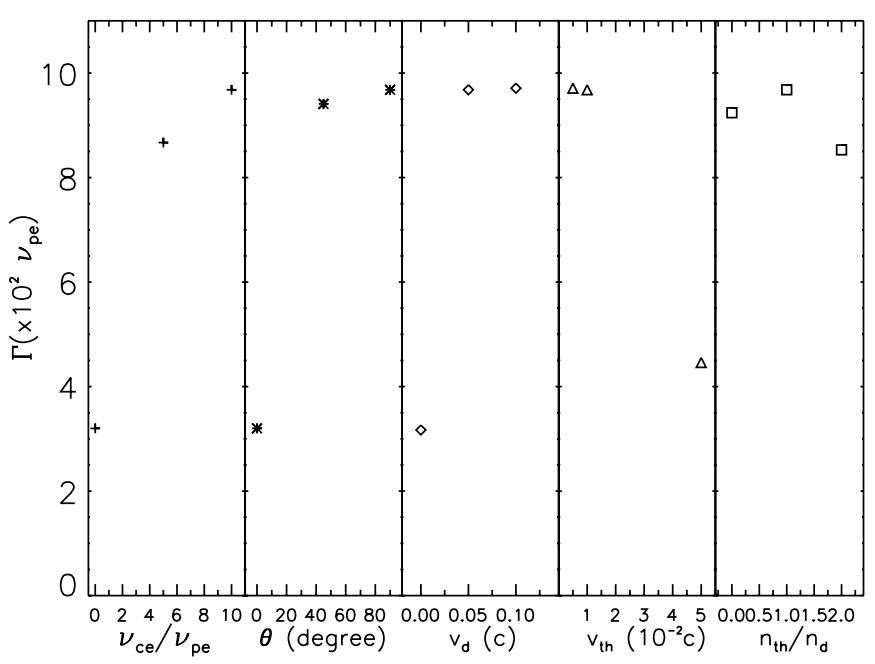

Figure 8. Maximum growth rate of the field energy in each simulation.

We clearly see many emission and absorption lines in Figure 9 that represent the EM field energy distribution at different frequencies. We find that the majority of the field energy is from frequencies $<80 v_{\mathrm{pe}}$ with bandwidth at half-maxima $\sim 6 v_{\mathrm{pe}}$.

\section{RESULTS II: $l=3$}

In order to see the interactions of the electrons with different initial velocity distributions, we perform another series of simulations with $l=3$. In this case, when $v_{\mathrm{d}}=0$, Equation (15) is a typical loss-cone velocity distribution. Note that in this section, we take $v_{\mathrm{d}}=0$ in the standard model to exclude the effect of the electron beam. We vary the ther- mal velocity of the electrons to see the effect of the temperature on the energy exchange and release (movies are available for the spatial and velocity evolution of the system at http://www.arm.ac.uk/highlights/2012/600/losscone/).

\subsection{Standard Model and Influence of Parameters in Loss-cone-driven ECM}

Figure 10 illustrates the EM field in the left panels, and in the right panel we have the thermal and drift energy of the electrons from the loss-cone-driven ECM. From this figure, we see that only a very small fraction of kinetic energy is converted to EM field energy if there is no external magnetic field or the losscone velocity distribution is along the direction parallel to the external magnetic field.

In other cases, an increase in any one of the parameters $B_{0}$, $\theta$, and $v_{\text {th }}^{\prime}$ leads to an increase in the induced EM field energy. Raising the temperature of the background electrons (i.e., the thermal level) can suppress the growth of the field energy. Since we only vary the number of background electrons in a very small range, it does not affect the field energy significantly. As seen from the left bottom panel in Figure 10, increasing the number of background electrons decreases the induced EM field energy. These results are consistent with the growth rate of the EM wave illustrated in Figure 11. From the right panels in Figure 10, we see that the drift energies are rapidly dissipated, eventually leading to irregular motion of the electrons in the external magnetic field.

The history of the degree of the polarization of the EM waves in each simulation is shown in Figure 12. The conditions for the circularly polarized EM waves are notable, ranging from (1) decreasing the magnetic field, (2) varying the angle 


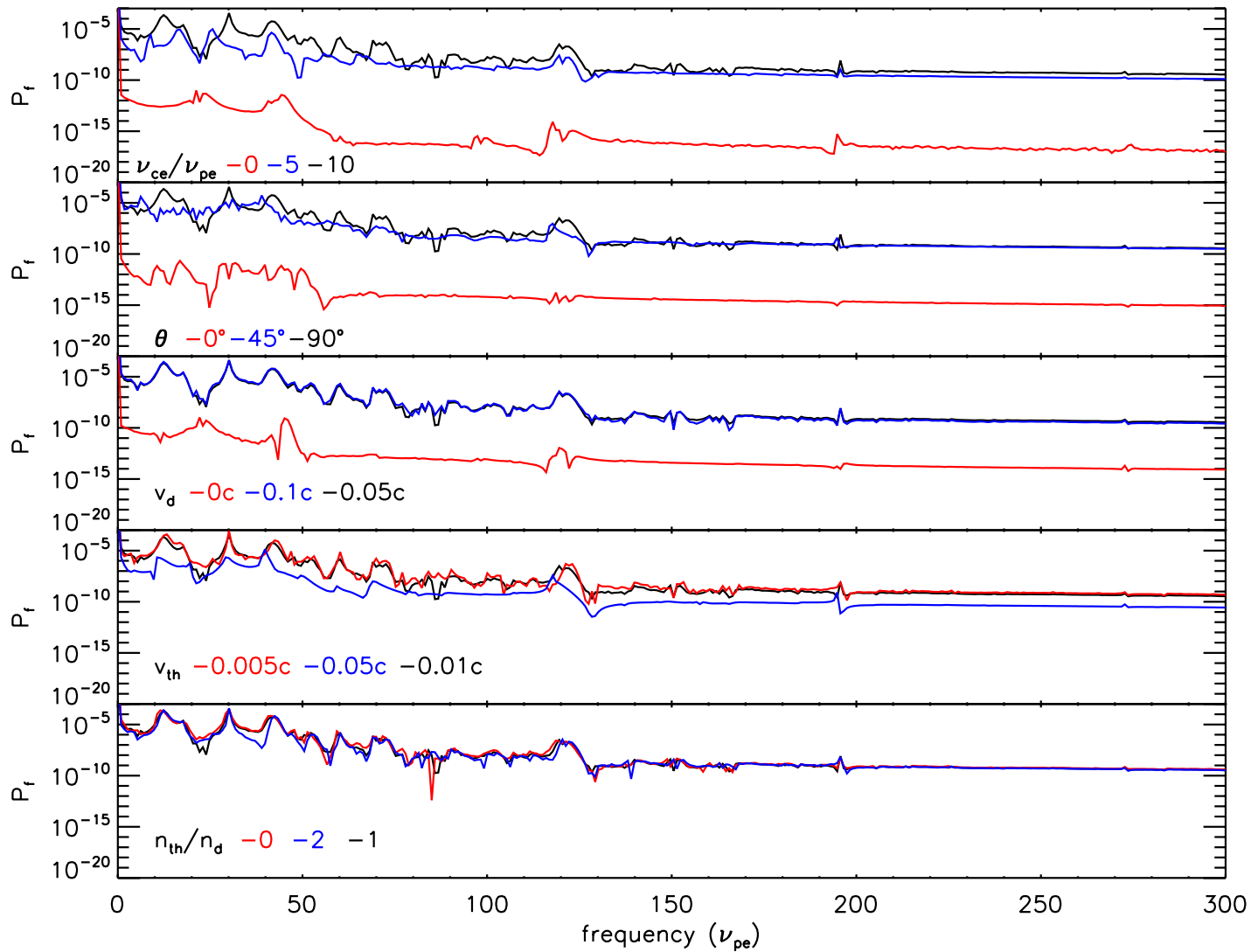

Figure 9. Same as Figure 7 but for the spectral energy density $\left(P_{f}\right)$ in each of the simulations in the non-relativistic beam-driven instability. (A color version of this figure is available in the online journal.)
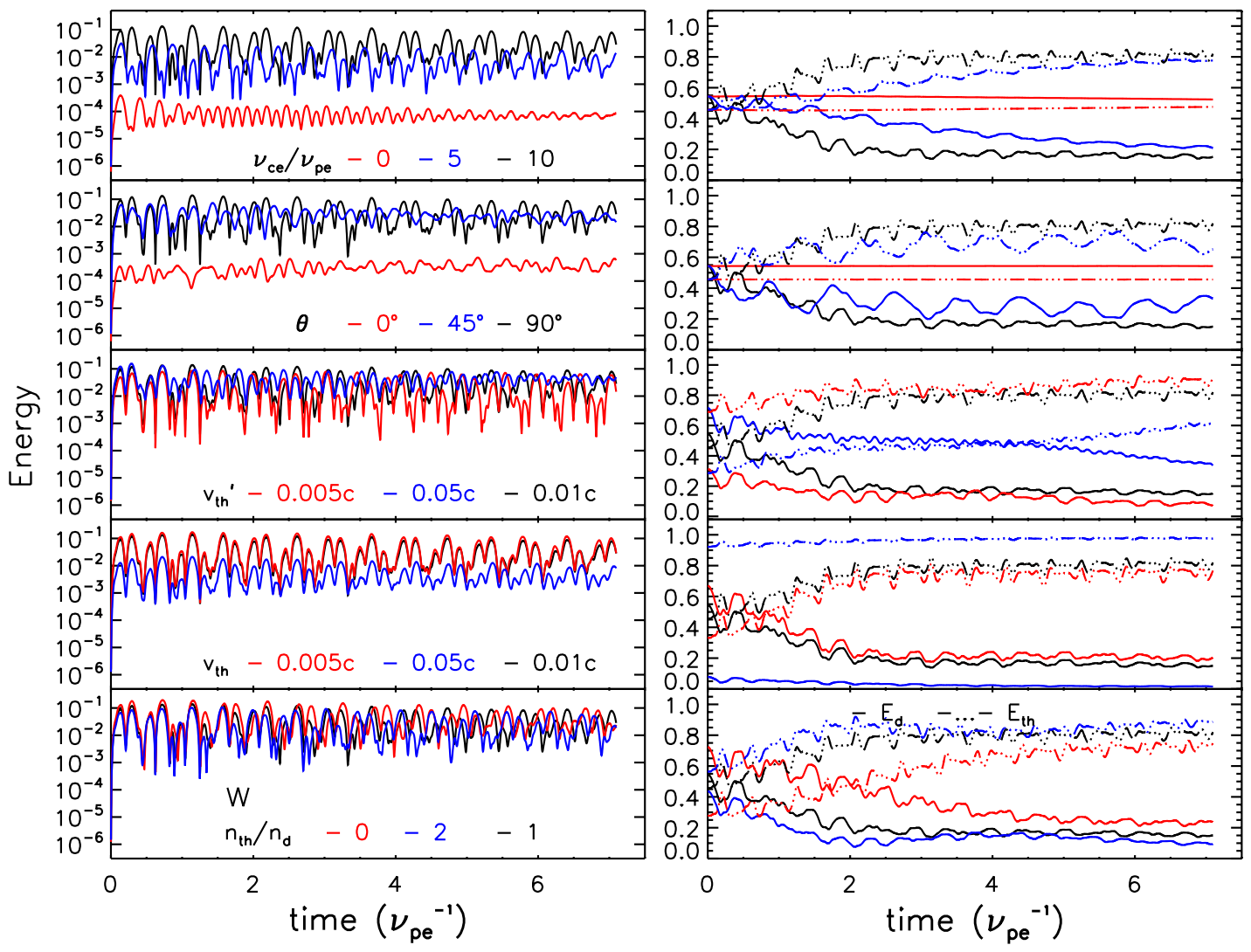

Figure 10. Same as Figure 6 but for the loss-cone-driven ECM.

(A color version of this figure is available in the online journal.) 


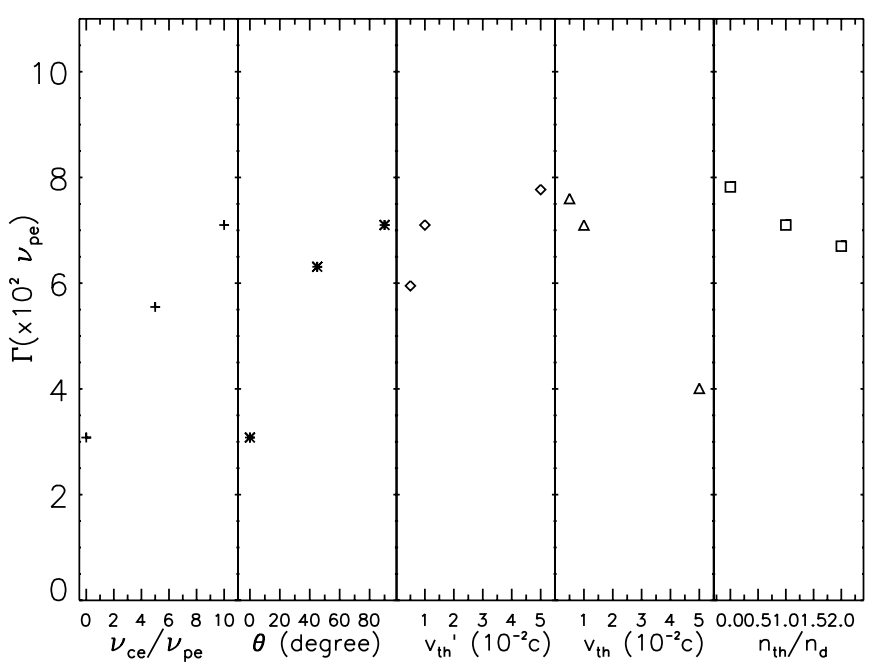

Figure 11. Maximum growth rate of the field energy in the loss-cone-driven ECM.

$\theta$ from perpendicular to non-perpendicular, to (3) increasing the thermal level of the background electrons. We suggest that these conditions may be associated with the circularly polarized components of the radio emission from UCDs.

\subsection{Spectrum}

The influence of the parameters on the spectrum is shown in Figure 13, in which we find that the magnetic field and the angel $\theta$ can affect the SED significantly, while the thermal effect and the number of background electrons only play a minor role. Some frequency bands are notable, e.g., from 10 to $70 v_{\text {pe }}$.

\subsection{Comparison between Beam-driven and Loss-cone-driven ECM}

Comparison between the different initial velocity distributions can help us understand the roles of the initial parameters in the process of releasing EM wave energy and the transfer between drift energy and thermal energy. Combining Figures 6 and 7 (beam-driven) and Figures 10 and 12 (loss-cone-driven), we see that

1. the existence of the drift kinetic energy of the electrons can be considered as a coherent current (which is necessary to generate the intense EM field energy), while the form of the initial velocity distribution is not important;

2. in order to efficiently obtain the intense EM field energy, the external magnetic field plays a crucial role. The angle between the magnetic field and the coherent current significantly affects the strength of the released EM field energy in a nonlinear relation (see Figures 8 and 11) and also the propagation direction of the EM waves;

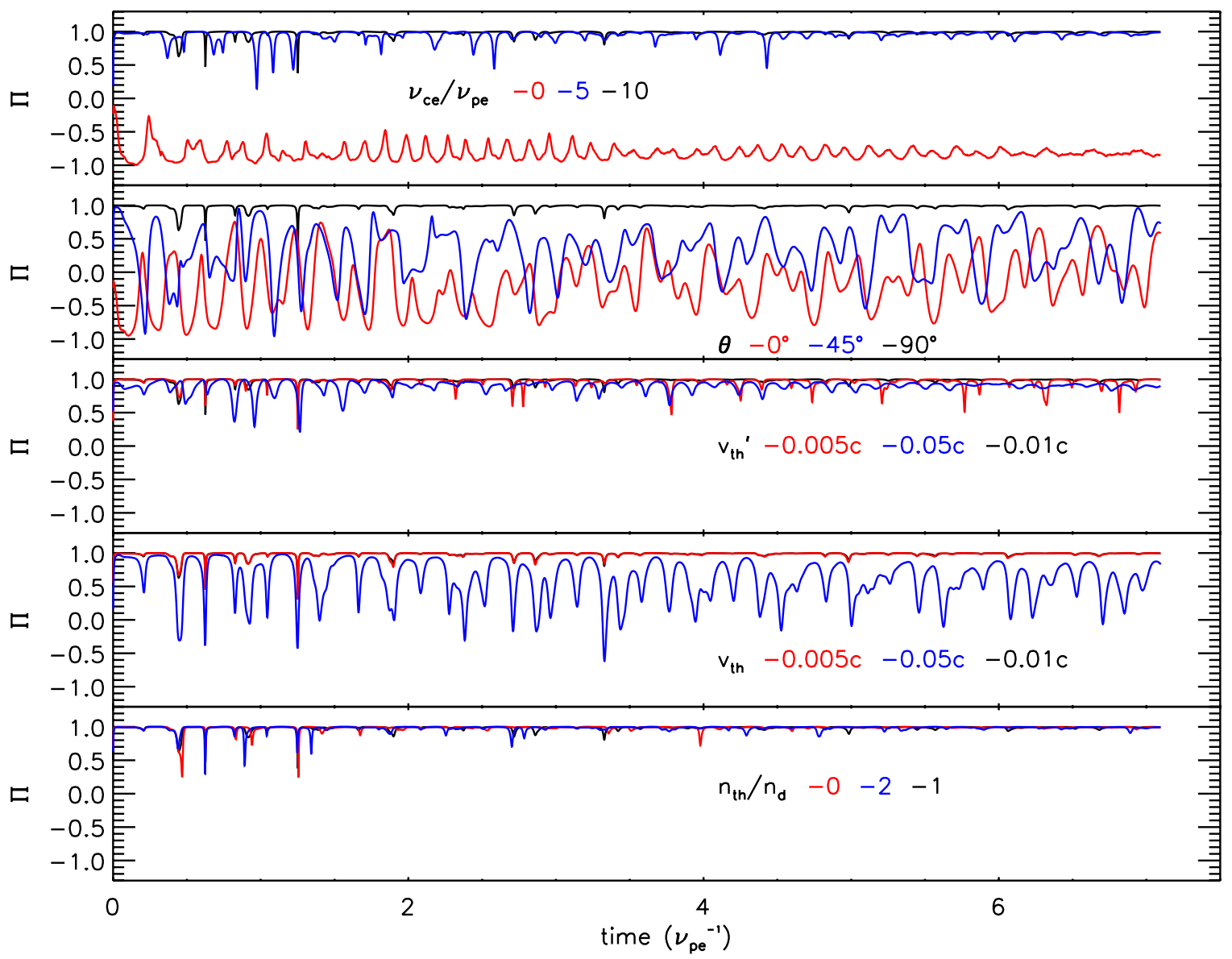

Figure 12. Time evolution of the degree of linear polarization in the loss-cone-driven ECM. Each panel is for one parameter, i.e., from top to bottom, $v_{\mathrm{ce}} / v_{\mathrm{pe}}, \theta, v_{\mathrm{d}}$, $v_{\text {th }}, n_{\text {th }} / n_{\mathrm{d}}$. Different colors in each panel are for different values of the parameters as shown in the panel.

(A color version of this figure is available in the online journal.) 


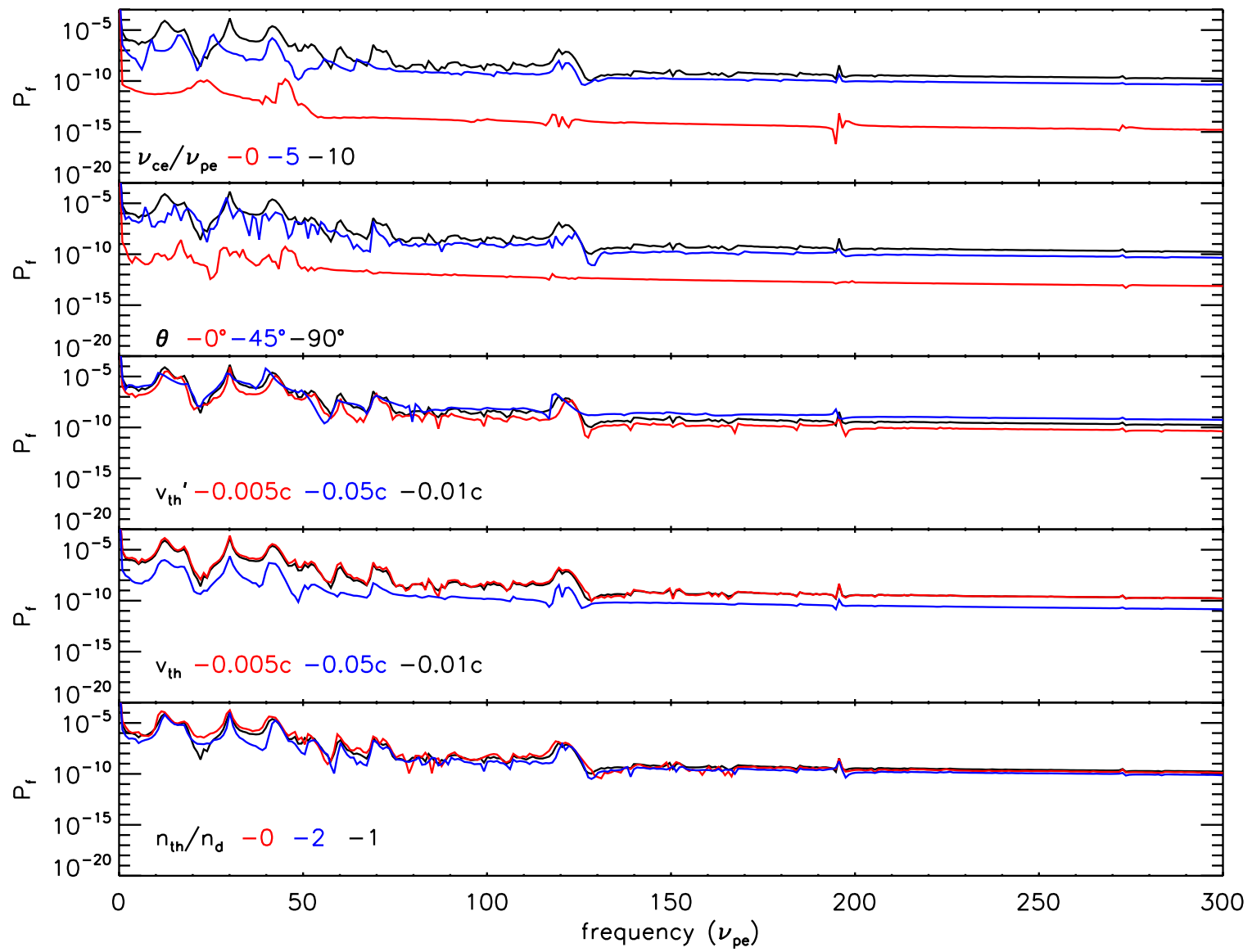

Figure 13. Same as Figure 9 but for the loss-cone-driven ECM.

(A color version of this figure is available in the online journal.)

3. pure thermal motion of the injected electrons in the external magnetic field may play a role in generating the EM waves (e.g., when $v_{\mathrm{d}}=0$ in the beam-driven ECM, the middle panel in Figure 6), while the thermal level of the background electrons mainly suppresses the generation of the EM waves.

The SED of the beam-driven and loss-cone-driven ECM indicates that all the parameters (except the number of background electrons in the present simulations) can affect the SED; however, certain harmonic frequency bands will appear if the coherent current is sufficiently strong, for example, see the region from 10 to $70 v_{\mathrm{pe}}$. There is a negligible signal in the very high frequency band $>100 v_{\text {pe }}$. Also, it seems that the SED weakly depends on the number of background electrons, but this needs further investigation since we do not vary the number of particles over a sufficiently wide range in the simulations.

\section{RELATIVISTIC BEAM-DRIVEN INSTABILITY}

In this section, we show the case where a relativistic electron beam moves in an external magnetic field. We take $l=0$ in Equation (15) and set the same standard parameter values as in Section 3 except here we take a larger value for the drift velocity of the electrons, i.e., $v_{\mathrm{d}}=0.98 c$ for the standard model; and $0.58 c$ and $0.78 c$ for the optional values. Furthermore, we only vary the thermal velocity of the background electrons, with values of $v_{\text {th }}=0.001 c, 0.01 c$ (standard value), and $0.1 c$.

\subsection{Influences of Parameters}

The history of the three kinds of energies, the polarization of the induced EM field energy, and their growth rates are illustrated in Figures 14-16. It is seen that the background electrons in the simulations have a negligible influence on the induced EM field energy and the linear polarization, while the thermal energy level of the background electrons can suppress the growth of the EM waves.

From the left panels in Figure 14, we find that the direction and values of the magnetic field and the drift velocity can significantly affect the induced EM field. The existence of the magnetic field is necessary in order to obtain the fast growth of the EM field energy, and the energy conversion efficiency seems to have a nonlinear relation with the magnetic field strength.

The right panels in Figure 14 illustrate the drift energy and the thermal energy of the electrons. If the magnetic field strength is sufficiently high to constrain the motion (or spatial position) of the injected electrons, the drift energy can be rapidly converted to the thermal energy in a timescale that is similar to the nonrelativistic case. If the magnetic field is not very strong, we see that it is possible for the system to still retain some residual drift energy when the electrons escape from the local region.

The influence of the parameters on the polarization in the relativistic case (Figure 15) is different from the non-relativistic case. The strength of the magnetic field in the present simulations affects the degree of the linear polarization. The existence of a strong magnetic field is essential to generate the linear polarization. The non-perpendicular angle between the magnetic 

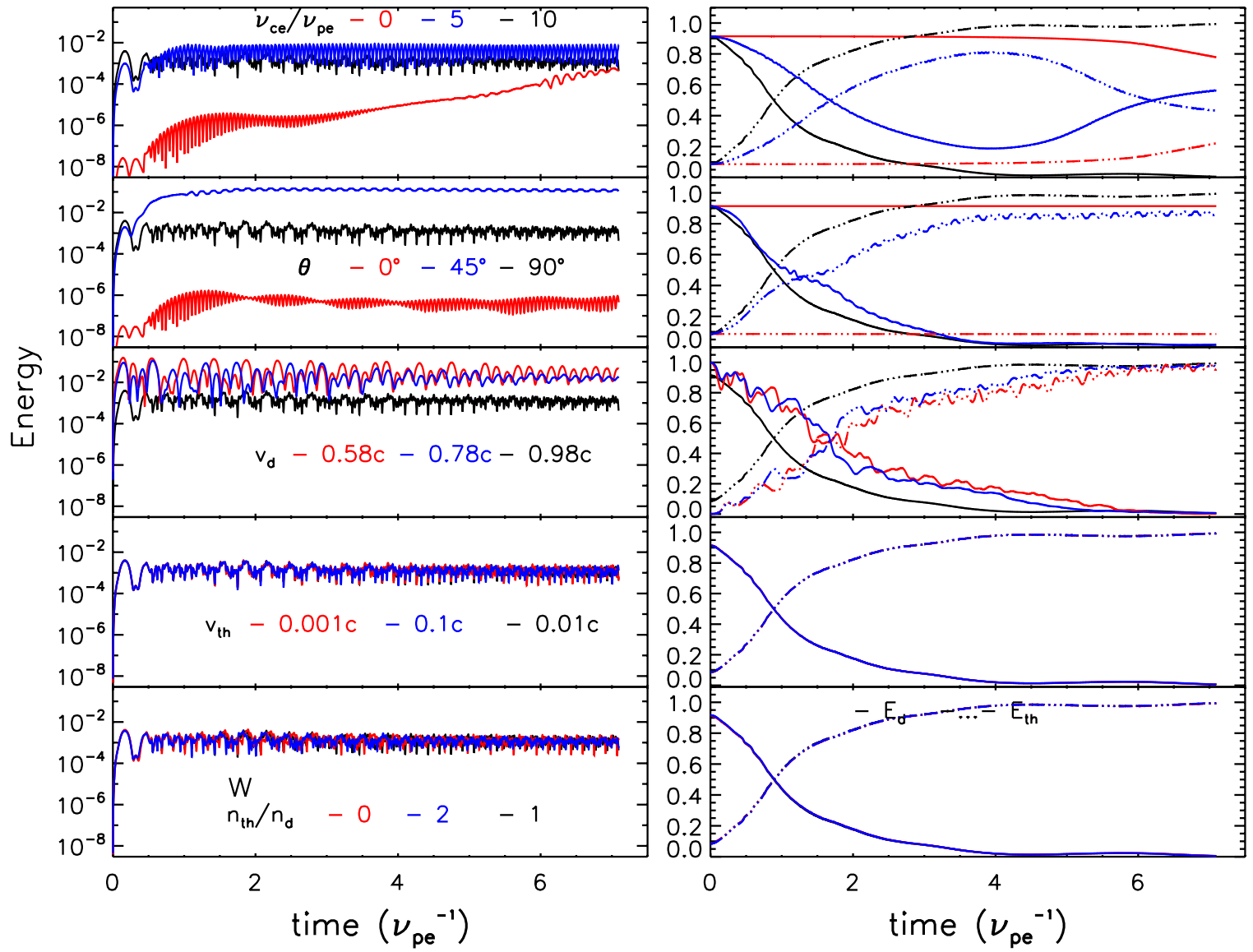

Figure 14. Same as Figure 6 but for the relativistic beam-driven instability.

(A color version of this figure is available in the online journal.)

field and the drift velocity plays an important role in the generation of the linear polarization. In fact, at high frequency, linear polarization becomes dominant in the relativistic case. The drift velocity, the thermal level of the background electrons, and the relative number of drift electrons and background electrons do not affect the polarization significantly in the present simulations. As shown in Figure 16, the growth rate of the EM field rapidly increases with the magnetic field strength and injection angle. However, it seems that in the relativistic case the increase of the drift velocity slightly decreases the growth of the EM field, perhaps because of a strong interaction between the induced EM field and the high-energy electron beam. The thermal level of the background electrons can suppress the growth of the EM field, and there is an ambiguous relation between the number of background electrons and the growth rate of the EM field in the present simulations.

The influence of the direction between the magnetic field and the drift velocity is very interesting since the maximum energy conversion efficiency occurs in the non-perpendicular injection, which differs from the non-relativistic case. In order to determine the direction where we can obtain the maximum energy conversion efficiency, we have done some extra computations by varying the direction of the magnetic field. Figure 17 illustrates the results in which we see that the maximum energy conversion efficiency occurs at $\sim 75^{\circ}$.

An interesting phenomenon in our simulations is that the velocity distribution of the beam electrons may evolve from an initially drifted Gaussian (or Gaussian-like) distribution to ring distribution (or incomplete-ring or spiral-ring or horse- shoe, e.g., see the standard models in both the relativistic case and non-relativistic case; a movie is available for the velocity evolution of the electron beam in the relativistic case at http://www.arm.ac.uk/highlights/2012/600/relativistic_beam/ relativistic_beam_velocity/) or spherical-shell distribution (or incomplete shell, e.g., if we change the angle between the magnetic field and the beam electron from perpendicular, e.g., $90^{\circ}$, to non-perpendicular, e.g., $45^{\circ}$ or $75^{\circ}$ ). This may imply that the ring and shell (or ring-like and shell-like) velocity distribution would have a common origin-beam distribution. The influence of the angle on the evolution of the velocity of electrons is mainly caused by the external magnetic field and the selfinduced EM fields. The spatial current induced by the motion of the charged particles plays an important role in the process, which initially causes the variation of a spatial magnetic field and time-dependent electric field, i.e., Equation (1). This electric field will accelerate or decelerate the electrons to deform the distribution values of the electron velocity.

\subsection{Spectrum}

The SED of the radiation in the relativistic electron beam apparently differs from that in the non-relativistic case. Figure 18 illustrates the SEDs in the relativistic case. In the standard model, we clearly see that the energy may not only distribute in the range of $10-100 v_{\text {pe }}$ but extend up to a frequency of $\sim 500 v_{\text {pe }}$ and some negligible signal at an even higher frequency harmonic.

For a single relativistic electron, the peak value of its synchrotron radiation may be at $v_{\text {peak }} \approx \gamma^{2} v_{\text {ce }} \sin \theta$ (Rybicki \& 


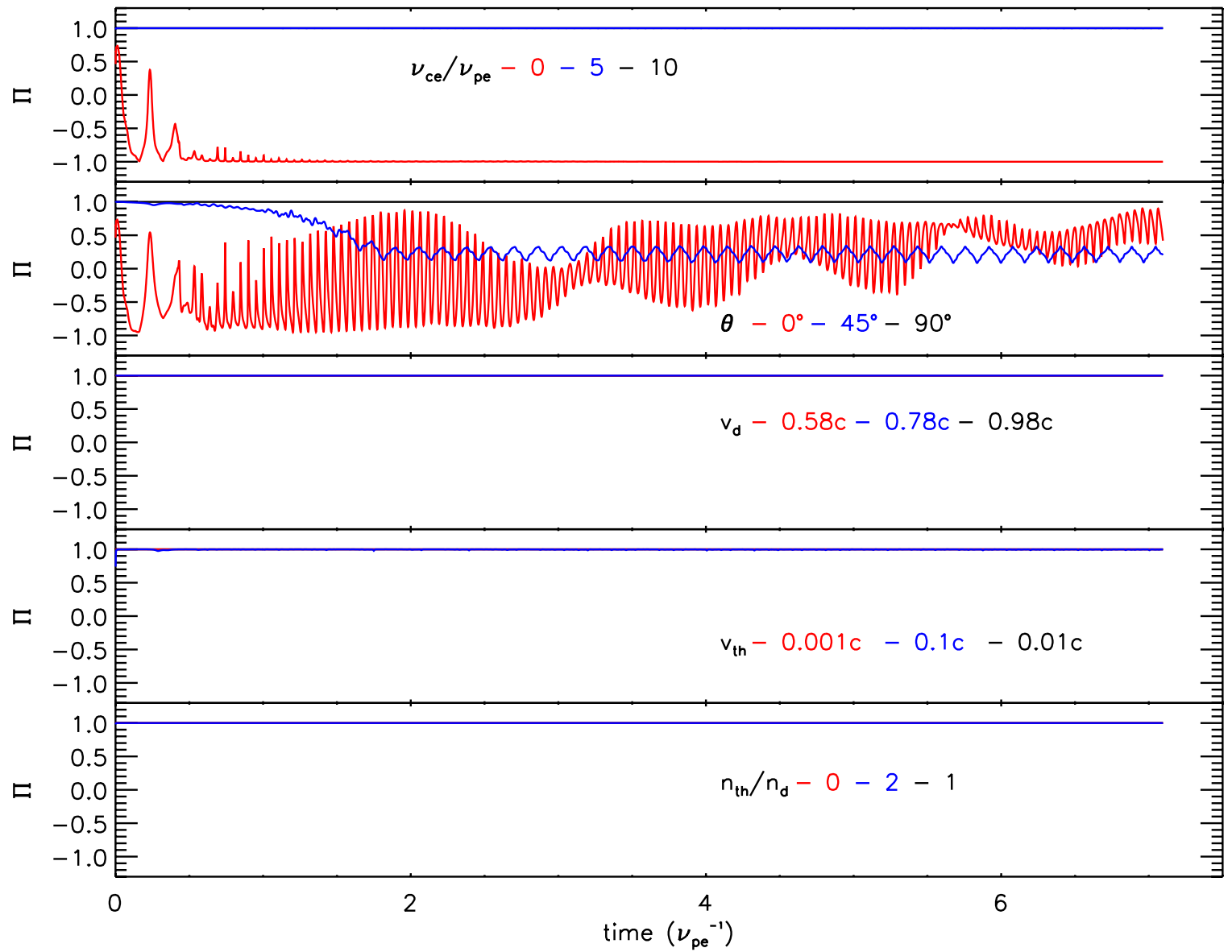

Figure 15. Evolution of the degree of linear polarization with time in the relativistic beam-driven instability. Each panel is for one parameter, i.e., from top to bottom, $v_{\mathrm{ce}} / v_{\mathrm{pe}}, \theta, v_{\mathrm{d}}, v_{\mathrm{th}}, n_{\mathrm{th}} / n_{\mathrm{d}}$. Different colors in each panel are for different values of the parameters as shown in the panel.

(A color version of this figure is available in the online journal.)

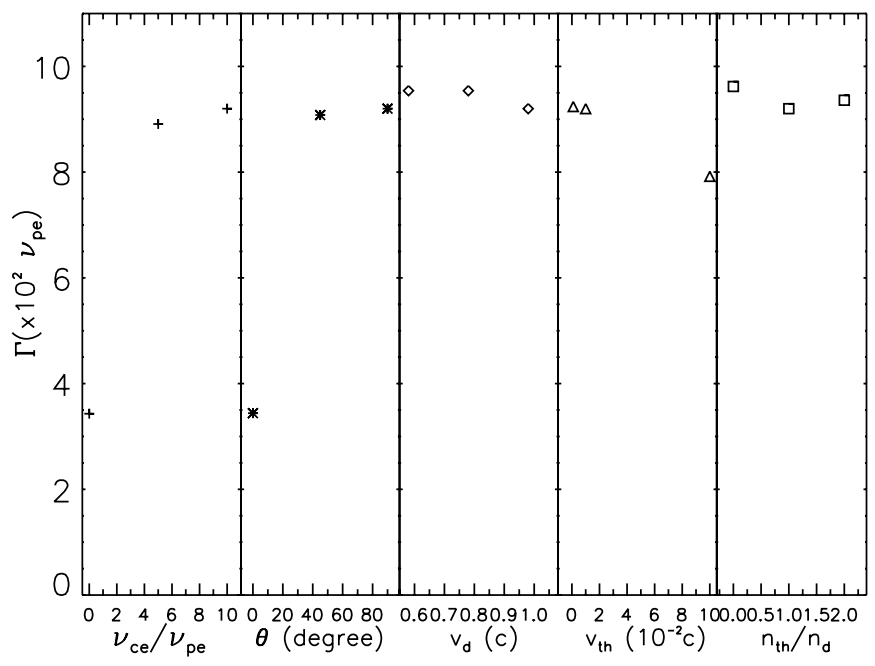

Figure 16. Maximum growth rate of the field energy in the relativistic beamdriven instability.

Lightman 1979). For a many-electron system, the frequencies where we can obtain emission lines are strongly associated with the distribution of the Lorentz factor $\gamma$. Figure 19 illustrates the distribution of $\gamma$ in different parameters. In the standard model, we find that when the instability reaches saturation, the distribution of $\gamma$ expands only a little in the high-energy part. However, when varying the angle $\theta$ from $90^{\circ}$ to $75^{\circ}$,

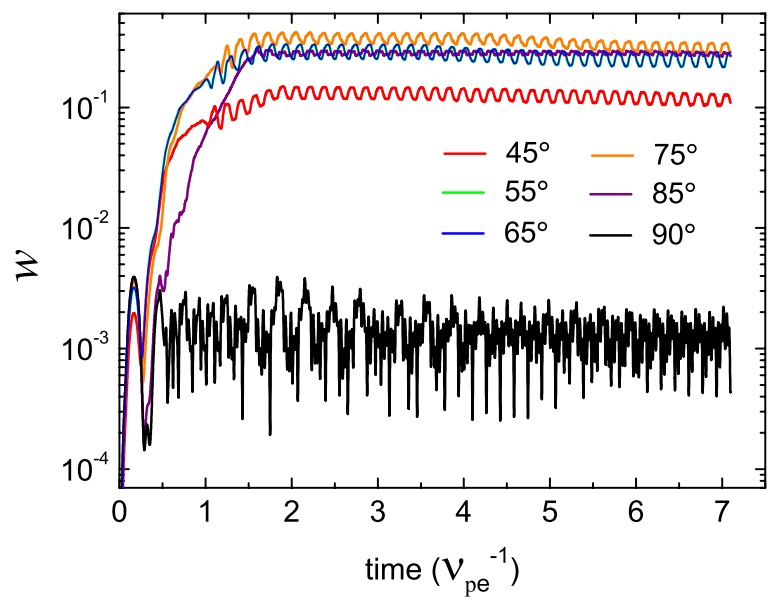

Figure 17. Energy history for the standard model with different angle between the external magnetic field and the injected velocity direction in the relativistic beam-driven instability, i.e., $45^{\circ}, 55^{\circ}, 65^{\circ}, 75^{\circ}, 85^{\circ}$, and $90^{\circ}$.

(A color version of this figure is available in the online journal.)

we see a distinct high-energy electron tail around $\gamma=9.5$, which corresponds to the kinetic energy $E_{i \mathrm{ek}}=(\gamma-1) m_{\mathrm{e}} c^{2} \approx$ 4.35 MeV. These high-energy electrons may contribute to possible X-ray emission from UCDs via thermal or non-thermal bremsstrahlung or even inverse Compton scattering. 


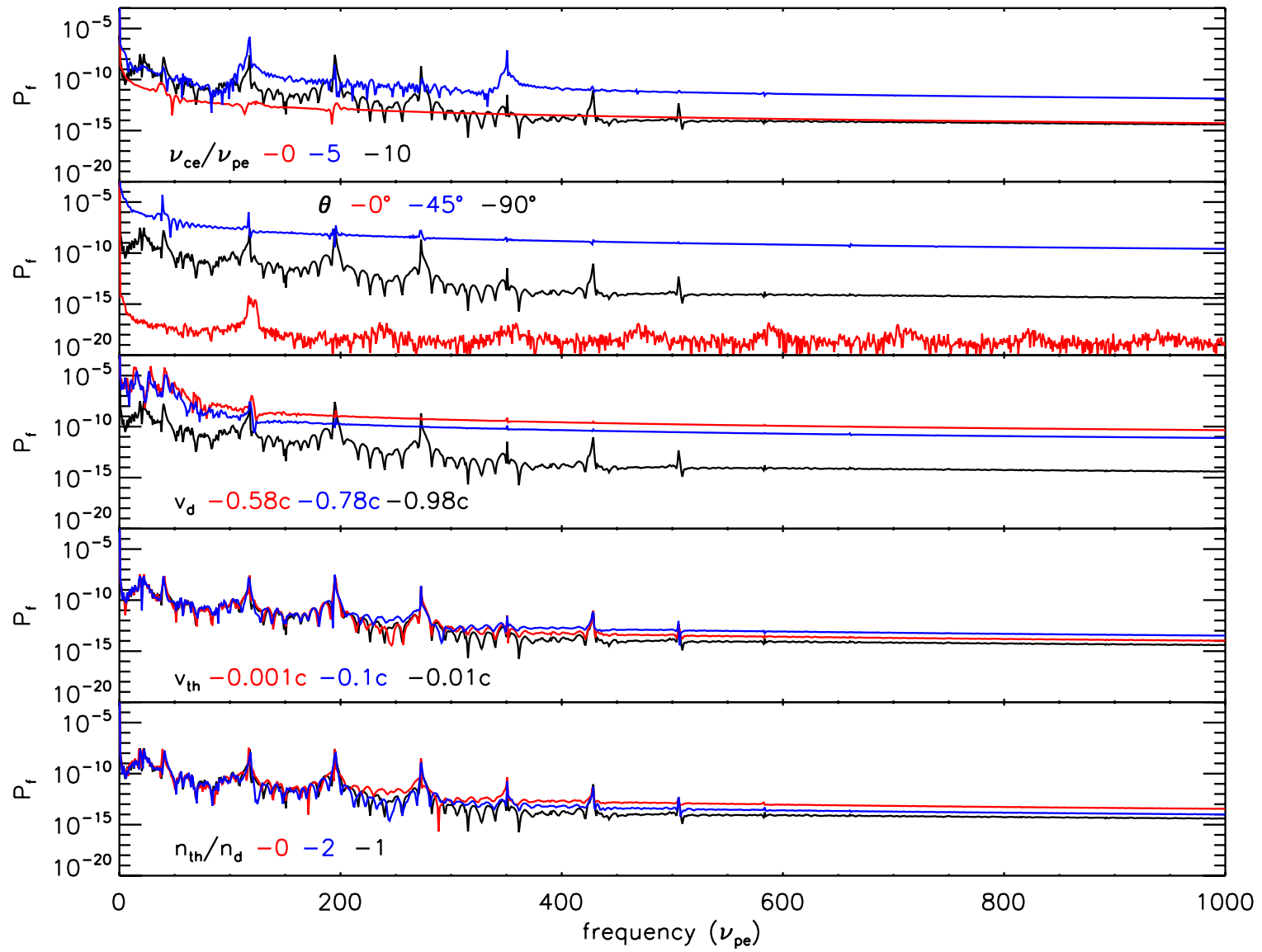

Figure 18. Same as Figure 9 but for the relativistic beam-driven instability.

(A color version of this figure is available in the online journal.)
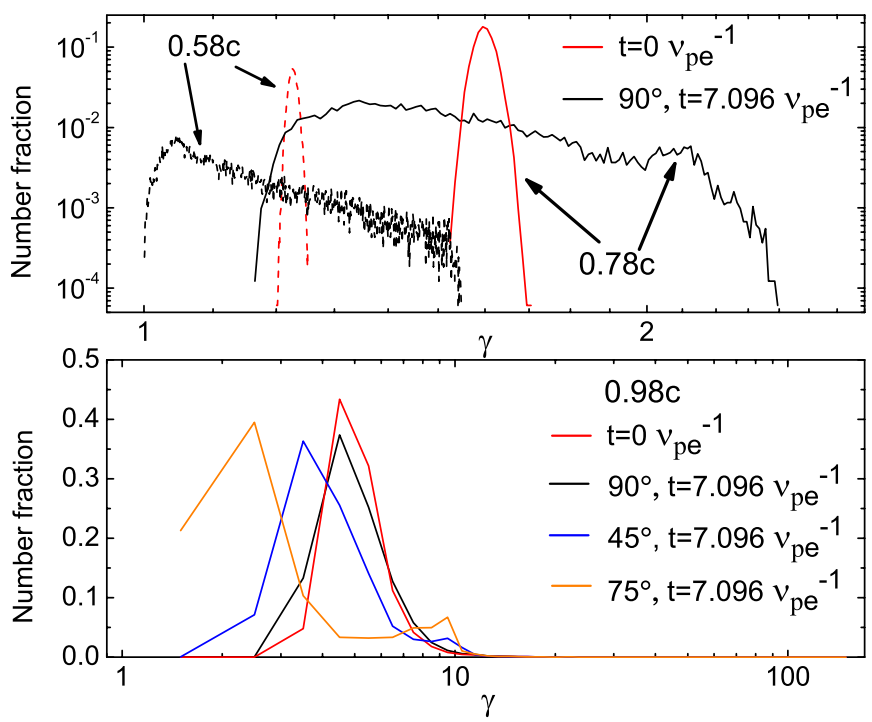

Figure 19. Energy distribution of the electrons in the relativistic beam-driven instability. Top panel is for the initially mildly relativistic electrons with drift velocity $0.58 c$ and $0.78 c$ and incident angle $90^{\circ}$; the bottom panel is for initially mildly relativistic electrons with fixed drift velocity $0.98 c$ and different incident angle, i.e., $45^{\circ}, 75^{\circ}$, and $90^{\circ}$. The number of electrons in each bin is normalized by the total number of injected electrons. The bin size is 0.001 for $0.58 c, 0.01$ for $0.78 c$, and 1 for $0.98 c$.

(A color version of this figure is available in the online journal.)

\section{COMPARISON WITH OBSERVATIONS AND OTHER STUDIES}

It is possible to approximately compare our simulation results with the observed radio spectrum from UCDs using some reference values of $v_{\mathrm{pe}}$ in Table 1. For example, if we assume that the observed radio emission from TVLM 513-46546 at $8.5 \mathrm{GHz}$ corresponds to the simulated peak frequency at $\sim 12 v_{\mathrm{pe}}$ in the case of $v_{\mathrm{ce}} / v_{\mathrm{pe}}=10$ (the strongest in the simulation) in the nonrelativistic beam-driven instability, then $v_{\mathrm{pe}}=0.708 \mathrm{GHz}$ and $v_{\mathrm{ce}}=7.08 \mathrm{GHz}$. Thus, the corresponding local plasma density is $\sim 6.2 \times 10^{9} \mathrm{~cm}^{-3}$ and the strength of the magnetic field is $\sim 2529 \mathrm{G}$. We may find some weaker signal at a high-frequency harmonic, such as $\sim 30 v_{\mathrm{pe}}=21.24 \mathrm{GHz}, \sim 42 v_{\mathrm{pe}}=29.74 \mathrm{GHz}$, $\sim 50 v_{\mathrm{pe}}=35.4 \mathrm{GHz}$.

In the relativistic case, the first significant signal appears at $\sim 20 v_{\mathrm{pe}}$ when $v_{\mathrm{ce}} / v_{\mathrm{pe}}=10$, which might correspond to the observed radio signal at $8.5 \mathrm{GHz}$. This gives $v_{\mathrm{pe}}=0.425 \mathrm{GHz}$ and $v_{\mathrm{ce}}=4.25 \mathrm{GHz}$. Thus, the corresponding local plasma density is $\sim 2.2 \times 10^{9} \mathrm{~cm}^{-3}$ and the strength of the magnetic field is $\sim 1518 \mathrm{G}$. We may find some weaker signal at a higher frequency harmonic, such as $\sim 40 v_{\mathrm{pe}}=17 \mathrm{GHz}, \sim 118 v_{\mathrm{pe}}=$ $50.15 \mathrm{GHz}, \sim 195 v_{\mathrm{pe}}=82.875 \mathrm{GHz}$, etc. This means the spectrum may extend to the extreme high frequency band or even far-infrared in the extremely relativistic case.

If we choose $v_{\mathrm{ce}} / v_{\mathrm{pe}}=5$ in the relativistic case and assume that the first significant signal appearing at $\sim 110 v_{\mathrm{pe}}$ corresponds 
to the observed radio signal at $8.5 \mathrm{GHz}$, it gives $v_{\mathrm{pe}}=$ $0.0773 \mathrm{GHz}$ and $v_{\mathrm{ce}}=0.386 \mathrm{GHz}$. Thus, the corresponding local plasma density is $\sim 7.4 \times 10^{7} \mathrm{~cm}^{-3}$ and the strength of the magnetic field is $\sim 138 \mathrm{G}$.

The above estimations of the properties of the emission region and the spectrum distributions in the present simulations are consistent with the analytical results in Dulk (1985) and Güdel (2002).

We do not draw conclusions on the values of plasma parameters in this paper because, first, many parameters are free in our simulations. Especially two of the key parameters, the local electron density and magnetic field, are ambiguous. Second, the present simulations are confined in a local microregion with no consideration of absorption and emission by other regions. The absorption and re-emission by the plasma in other regions need to be further investigated. The radio spectrum can be affected by the large-scale structure of a magnetic field (Kuznetsov et al. 2012). A combination of observations at other wavelengths will help us determine the configuration of the magnetic field and the plasma environment and thus entirely understand the magnetosphere and atmosphere of UCDs.

The simulation results of the radiopulses from one of the radioactive UCDs in Yu et al. (2011) show that the losscone-driven ECM in Aschwanden (1990a) can result in the release of $\sim 0.5 \%$ of the kinetic energy, which therefore can generate a strong radio flux of up to a few mJy. This depends on other parameters, for instance, the local plasma density. By comparison, the non-relativistic beam-driven ECM in the present model can release $\sim 2 \%$ of the kinetic energy when the process reaches saturation, which is about four times higher than the results in the model of Aschwanden (1990a).

Our simulation results are also comparable with the studies for auroral kilometric radiation regions. Pritchett et al. (2002) investigated the auroral kilometric radiation source cavity using two-dimensional particle-in-cell simulations. They found the energy conversion efficiency to be $\sim 1 \%-5 \%$, depending on the velocity of the hot electrons $(\sim 1-10 \mathrm{keV})$. The timescale of the radiation bursts is $\sim 0.5 \mathrm{~ms}$, which is probably associated with the local plasma density $\left(\lesssim 1 \mathrm{~cm}^{-3}\right.$; Strangeway et al. 1998; Perraut et al. 1990; Calvert 1981) and the weak magnetic field. The energy conversion efficiency in our results is in line with that in their results in the non-relativistic cases. The spectra obtained in the present simulations are also in good agreement with the previous results (Melrose \& Dulk 1982; Aschwanden 1990a; Pritchett 1986, 1984)—-the waves are produced mainly near the electron cyclotron frequency.

However, certain differences exist between our simulation results and others. First, in our simulations, we can see the different oscillation modes from the energy time history that reflect the interaction between the particles and the induced electric field. Second, we find that the energy conversion efficiency can be influenced significantly by the drift velocity of the particles and the angle between the drift velocity and the magnetic field, which is perhaps caused by the self-induced EM damping. The initial conditions and the technique to solve Maxwell's equations in our simulations and others are also different. We considered a spatially localized electron population with a beam or beam-like velocity distribution. In contrast, previous works considered ring-like, horseshoe, and Dory-Guest-Harris distributions (Pritchett et al. 2002; Pritchett 1986,1984 ) and a larger space structure for the magnetic field. The time advancement of Maxwell's equations was performed in Fourier space in Pritchett et al. (2002). Instead, we used a staggered leap-frog method to solve the equations in the time domain with the positions and velocities of the electrons generated by a Monte Carlo method.

Recent computer simulations and experimental laboratory work by Cairns et al. (2011) and Vorgul et al. (2011) show that in the non-relativistic (or weakly relativistic) case the energy conversion efficiency is $1 \%-2 \%$, which is consistent with our simulations. However, this energy conversion efficiency varies significantly in our relativistic case, depending on the parameters, e.g., the angle, the drift velocity, and the strength of the external magnetic field. It may be mainly caused by the effect of synchrotron radiation.

\section{SUMMARY AND CONCLUSIONS}

In this paper, we present the numerical simulations for electron-beam-driven and loss-cone-driven ECM with different plasma parameters and different magnetic field strengths. We find that the beam-driven ECM can be an effective mechanism to release EM waves and heat the surrounding plasmas. From the diffusion process of the electrons in velocity space and the energy distribution (see Figures 3 and 19), a high-energy tail of the electrons may be rapidly developed along the direction near perpendicular to the magnetic field, which can eventually evolve to moderately or strongly relativistic electrons, depending on the initial energy of the electron current, and contribute to gyrosynchrotron or synchrotron radiation. This may lead to the appearance of a radio continuum and the deformation of the SED. Also, these high-energy electrons may be important to generate X-ray emission.

The computation of the degree of polarization indicates that the thermal level of the electrons can significantly affect the degree of the circular or linear components of the observed radio waves. In the case of the beam-driven ECM, the angle between the direction of the magnetic field and the injection direction of the injected electrons is another crucial factor to affect the degree of circular polarization in the radio waves.

The SEDs of the radio waves depend weakly on the form of the velocity distribution of the electrons in the present simulations. The existence of the external magnetic field and the angle between the direction of the magnetic field and the moving direction of the electron current can significantly affect the SED. Certain frequency bands, e.g., $10-70 v_{\text {pe }}$ in the non-relativistic case and $10-600 v_{\text {pe }}$ in the relativistic case, may appear, which increases the difficulty of finding the fundamental cyclotron frequency in the observed radio frequencies. It is, however, possible that magnetic field inhomogeneities may smooth out some of these bands, thus producing a continuous spectrum. In order to determine the plasma frequency and the cyclotron frequency, wide frequency bands should be covered by future radio observations.

The present study is limited in that only two-dimensional EM simulations are performed, with no consideration of the change of the magnetic field configuration and the influence of gravity. We will continue to develop the simulations to match the plasma environment and the magnetic topology on UCDs in order to understand their radio emission.

Research at the Armagh Observatory is grant-aided by the N. Ireland Department of Culture, Arts, and Leisure. We also thank the Leverhulme Trust for their support of this project. A.A. gratefully acknowledges the support of the Bulgarian National Science Fund (Grant No. DDVU02/40-2010). A.L.M. thanks STFC for support through a Rolling Grant. S.Y. thanks the 9th 
international space simulation school for the tutorial courses and Professor Y. Omura for discussions. S.Y. also thanks Dr. J. McDonald in NUI, Galway for discussions. We thank the referee for his/her helpful and constructive comments.

\section{REFERENCES}

Antonova, A., Doyle, J. G., Hallinan, G., Bourke, S., \& Golden, A. 2008, A\&A, 487,317

Antonova, A., Doyle, J. G., Hallinan, G., Golden, A., \& Koen, C. 2007, A\&A, 472,257

Aschwanden, M. J. 1990a, A\&AS, 85, 1141

Aschwanden, M. J. 1990b, A\&A, 237, 512

Begelman, M. C., Ergun, R. E., \& Rees, M. J. 2005, ApJ, 625, 51

Berger, E. 2002, ApJ, 572, 503

Berger, E. 2006, ApJ, 648, 629

Berger, E., Ball, S., Becker, K. M., et al. 2001, Nature, 410, 338

Berger, E., Basri, G., Fleming, T. A., et al. 2010, ApJ, 709, 332

Berger, E., Rutledge, R. E., Phan-Bao, N., et al. 2009, ApJ, 695, 310

Berger, E., Rutledge, R. E., Reid, I. N., et al. 2005, ApJ, 627, 960

Birdsall, C. K., \& Langdon, A. B. 1985, Plasma Physics via Computer Simulation (New York: McGraw-Hill)

Burgasser, A. J., \& Putman, M. E. 2005, ApJ, 626, 486

Cairns, R. A., Vorgul, I., Bingham, R., et al. 2011, Phys. Plasmas, 18, 022902

Calvert, W. 1981, Geophys. Res. Lett., 8, 919

Chabrier, G., \& Küker, M. 2006, A\&A, 446, 1027

Chiu, Y. T., \& Schulz, M. 1978, J. Geophys. Res., 83, 629

Doyle, J. G., Antonova, A., Marsh, M. S., et al. 2010, A\&A, 524, A15

Dulk, G. A. 1985, ARA\&A, 23, 169

Durney, B. R., De Young, D. S., \& Roxburgh, I. W. 1993, Sol. Phys., 145, 207

Ergun, R. E., Carlson, C. W., McFadden, J. P., et al. 2000, ApJ, 538, 456

Fleishman, G. D., Gary, D. E., \& Nita, G. M. 2003, ApJ, 593, 571

Grießmeier, J., Zarka, P., \& Spreeuw, H. 2007, A\&A, 475, 359

Güdel, M. 2002, ARA\&A, 40, 217

Hallinan, G., Antonova, A., Doyle, J. G., et al. 2006, ApJ, 653, 690

Hallinan, G., Bourke, S., Lane, C., et al. 2007, ApJ, 663, L25

Helling, C., Jardine, M., Witte, S., \& Diver, D. A. 2011, ApJ, 727, 4

Hess, S., Mottez, F., \& Zarka, P. 2007a, J. Geophys. Res., 112, 11212

Hess, S., Zarka, P., \& Mottez, F. 2007b, Planet. Space Sci., 55, 89
Hockney, R. W., \& Eastwood, J. W. 1981, Computer Simulation Using Particles (New York: McGraw-Hill)

Jaeger, T. R., Osten, R. A., Lazio, T. J., Kassim, N., \& Mutel, R. L. 2011, AJ, 142,189

Jardine, M., \& Cameron, A. C. 2008, A\&A, 490, 843

Kuznetsov, A. A., Doyle, J. G., Yu, S., et al. 2012, ApJ, 746, 99

Lamy, L., Schippers, P., Zarka, P., et al. 2010, Geophys. Res. Lett., 37, 12104

Lo, K. K., Bray, J. D., Hobbs, G., et al. 2012, MNRAS, 421, 3316

Louarn, P., Le Queau, D., \& Roux, A. 1986, A\&A, 165, 211

McLean, M., Berger, E., \& Reiners, A. 2012, ApJ, 746, 23

Melrose, D. B., \& Dulk, G. A. 1982, ApJ, 259, 844

Melrose, D. B., \& Dulk, G. A. 1984, ApJ, 282, 308

Omura, Y. 2005, in Proc. 7th International School for Space Simulation

Omura, Y., \& Matsumoto, H. 1993, in Technical Guide to One-dimensional Electromagnetic Particle Code, ed. H. Matsumoto \& Y. Omura (Computer Space Plasma Physics; Tokyo), 21

Osten, R. A., Phan-Bao, N., Hawley, S. L., Reid, I. N., \& Ojha, R. 2009, ApJ, 700,1750

Parker, E. N. 1955, ApJ, 122, 293

Perraut, S., de Feraudy, H., Roux, A., Décréau, P. M. E., \& Paris, J. 1990, J. Geophys. Res., 95, 5997

Pritchett, P. L. 1984, J. Geophys. Res., 89, 8957

Pritchett, P. L. 1986, J. Geophys. Res., 91, 13569

Pritchett, P. L., \& Strangeway, R. J. 1985, J. Geophys. Res., 90, 9650

Pritchett, P. L., Strangeway, R. J., Ergun, R. E., \& Carlson, C. W. 2002, J. Geophys. Res. (Space Phys.), 107, 1437

Ravi, V., Hallinan, G., Hobbs, G., \& Champion, D. J. 2011, ApJ, 735, L2

Rybicki, G. B., \& Lightman, A. P. 1979, Radiative Processes in Astrophysics (New York: Wiley-Interscience), 393

Strangeway, R. J., Kepko, L., Elphic, R. C., et al. 1998, Geophys. Res. Lett., 25, 2065

Treumann, R. A. 2006, A\&AR, 13, 229

Villasenor, J., \& Buneman, O. 1992, Comput. Phys. Commun., 69, 306

Vorgul, I., Kellett, B. J., Cairns, R. A., et al. 2011, Phys. Plasmas, 18, 056501

Whelan, E. T., Ray, T. P., Randich, S., et al. 2007, ApJ, 659, L45

Wu, C. S., \& Lee, L. C. 1979, ApJ, 230, 621

Yu, S., Hallinan, G., Doyle, J. G., et al. 2011, A\&A, 525, A39

Zarka, P. 1998, J. Geophys. Res., 103, 20159

Zarka, P. 2004, Adv. Space Res., 33, 2045

Zarka, P. 2007, Planet. Space Sci., 55, 598 